\title{
Normal forms of conditional knowledge bases respecting system P-entailments and signature renamings
}

\author{
Christoph Beierle ${ }^{1}$. Jonas Haldimann ${ }^{1}$
}

Accepted: 22 April 2021 /Published online: 17 August 2021

(C) The Author(s) 2021

\begin{abstract}
Conditionals are defeasible rules of the form If $A$ then usually $B$, and they play a central role in many approaches to nonmonotonic reasoning. Normal forms of conditional knowledge bases consisting of a set of such conditionals are useful to create, process, and compare the knowledge represented by them. In this article, we propose several new normal forms for conditional knowledge bases. Compared to the previously introduced antecedent normal form, the reduced antecedent normal form (RANF) represents conditional knowledge with significantly fewer conditionals by taking nonmonotonic entailments licenced by system $\mathrm{P}$ into account. The renaming normal form $(\rho N F)$ addresses equivalences among conditional knowledge bases induced by renamings of the underlying signature. Combining the concept of renaming normal form with other normal forms yields the renaming antecedent normal form $(\rho \mathrm{ANF})$ and the renaming reduced antecedent normal form ( $\rho \mathrm{RANF})$. For all newly introduced normal forms, we show their key properties regarding, existence, uniqueness, model equivalence, and inferential equivalence, and we develop algorithms transforming every conditional knowledge base into an equivalent knowledge base being in the respective normal form. For the most succinct normal form, the $\rho$ RANF, we present an algorithm $K B^{\rho r a}$ systematically generating knowledge bases over a given signature in $\rho$ RANF. We show that the generated knowledge bases are consistent, pairwise not antecedentwise equivalent, and pairwise not equivalent under signature renaming. Furthermore, the algorithm is complete in the sense that, when taking signature renamings and model equivalence into account, every consistent knowledge base is generated. Observing that normalizing the set of all knowledge bases over a signature $\Sigma$ to $\rho$ RANF yields exactly the same result as $K B^{\rho r a}(\Sigma)$, highlights the interrelationship between normal form transformations on the one hand and systematically generating knowledge bases in normal form on the other hand.
\end{abstract}

Christoph Beierle

christoph.beierle@fernuni-hagen.de

Jonas Haldimann

jonas.haldimann@fernuni-hagen.de

1 FernUniversität in Hagen, 58084 Hagen, Germany 
Keywords Conditional knowledge base - Signature renaming - Inferential equivalence · Renaming normal form $\rho \mathrm{NF}$. Antecedent normal form ANF · Reduced antecedent normal form RANF $\cdot \rho \mathrm{ANF} \cdot \rho \mathrm{RANF} \cdot \mathrm{Knowledge}$ base generation

Mathematics Subject Classification (2010) 68T27 · 68T30 · 68T37

\section{Introduction}

For describing objects, situations, relationships, problems, solutions, etc., in a formal representation, adequacy, expressivity, and manageability of the used representation language are of crucial importance. This typically leads to many possibilities to express something in syntactically different, albeit semantically equivalent ways. On the other hand, the idea of normal forms is to reduce this redundancy by focussing on some standardized way of representation. Besides supporting standardized representations, benefits of normal forms include easier comparisons, avoidance of notorious borderline cases, fewer exceptions and thus clearer algorithms, and easier organization of proofs. Regarding the cost of normal forms, it has to be noted, though, that some machine-oriented normal forms, like the clause form of a formula in first-order predicate logic [1], may reduce human readability. Normal forms have been studied and employed extensively e.g. in logic formalisms or in automated theorem proving [2].

In this article, we deal with normal forms of conditional knowledge bases. A conditional knowledge base is a set of conditionals of the form $(B \mid A)$ representing a defeasible rule If $A$ then usually $B$. Such conditionals play a central role in nonmonotonic reasoning, and different semantic approaches have been defined for them (cf. [3-13]). The use of conditionals can be illustrated by the following example that models knowledge about electric cars.

Example 1 ( $\mathcal{R}_{\text {car }}$ [14]) Let $\Sigma_{c a r}=\{c, e, f\}$ be an alphabet where $c$ indicates whether something is a car, $e$ indicates whether something is an electric car (e-car), and $f$ indicates whether something needs fossil fuel.

The knowlegde base $\mathcal{R}_{\text {car }}$ contains the following nine conditionals expressing knowledge about e-cars:

$(f \mid c)$
$(\bar{f} \mid e)$
$(c \mid e)$
$(e \mid e \bar{f})$
$(e \mid e \bar{f})$
$(e \bar{f} \mid e)$
$(e \bar{f} \mid e)$
$(\bar{e} \mid \top)$
$(c f \vee \bar{c} f \mid c e \vee c \bar{e})$

"Usually cars need fossil fuel."

"Usually e-cars do not need fossil fuel."

"E-cars usually are cars."

"E-cars that do not need fossil fuel usually are e-cars."

"E-cars not needing fossil fuel usually are e-cars."

"E-cars usually are e-cars that do not need fossil fuel."

"E-cars usually are e-cars not needing fossil fuel."

"Usually things are not e-cars."

"Things that are cars and e-cars or cars but not e-cars are cars that need fossil fuel or are not cars but need fossil fuel."

Normal forms of conditional knowledge bases have been investigated in, e.g., [14-17].

Here, we will propose four new normal forms for conditional knowledge bases. The reduced antecedent normal form (RANF) extends the notion of antecedent normal form (ANF) introduced in [17] by taking non-monotonic entailments sanctioned by system P [7] into account. 
For classical logics, the motto "Truth is invariant under the change of notation" has been studied extensively, in particular in the framework of institutions [18]. Less attention has been paid to the aspect of change of notation in defeasible logics. In this work, we also address equivalences and transformations among conditional knowledge bases that take renamings of the underlying signature into account. The renaming normal form $(\rho N F)$ respects such signature renamings. Thus, while the RANF takes the semantic notion of model equivalence into account, the $\rho \mathrm{NF}$ refers to renaming equivalence on the syntactic level. We provide both a transformation system converting a conditional knowledge base into a model equivalent knowledge base in RANF as well as an algorithm to transform a knowledge base into a renaming equivalent knowledge base in $\rho$ NF.

Combining the normal forms ANF and RANF with $\rho$ NF yields the renaming antecedent normal form ( $\rho \mathrm{ANF}$ ) and the renaming reduced antecedent normal form ( $\rho \mathrm{RANF})$. By combining also the corresponding normal form transformations, we obtain algorithms transforming every knowledge base $\mathcal{R}$ into an equivalent knowledge base $\mathcal{R}^{\prime}$ that is in $\rho$ ANF or $\rho$ RANF, respectively.

For all introduced normal forms, we study and prove their key properties regarding, e.g., existence and uniqueness of normal forms, model equivalence, and inferential equivalence. For all corresponding normal form algorithms, we show their main characteristics, including termination, soundness, and completeness.

The framework we propose provides us with an automatic and powerful tool for checking whether two different knowledge bases induce the same set of entailments when taking both renamings and system $\mathrm{P}$ inferences into account. To illustrate this aspect, consider another example of a knowledge base.

Example $2\left(\mathcal{R}_{b c f}\right)$ Given the alphabet $\Sigma_{b c f}=\{b, c, f\}$, the knowledge base $\mathcal{R}_{b c f}$ consists of four conditionals:

$$
\mathcal{R}_{b c f}=\{(b \vee \bar{f} \mid f),(\bar{b} f \vee c \mid b),(b \bar{f} \vee \bar{c} \mid f),(\bar{f} \mid b \vee \bar{b} \vee f)\} .
$$

At first sight, the two knowledge bases $\mathcal{R}_{c a r}$ and $\mathcal{R}_{b c f}$ do not look very similar, but exhibit quite a few differences. However, considering renamings and system $\mathrm{P}$ inferences, both $\mathcal{R}_{c a r}$ and $\mathcal{R}_{b c f}$ are actually inferentially equivalent. Using our concepts and algorithms, this inferential equivalence is revealed easily by converting both $\mathcal{R}_{c a r}$ and $\mathcal{R}_{b c f}$ to $\rho$ RANF over a common signature and observing that their normal forms are syntactically identical.

In addition to providing methods for transforming a knowledge base to a normal form, we also address the problem of generating normal form knowledge bases. Here, we focus on the most succinct normal form, the $\rho$ RANF. We present an algorithm $K B^{\rho r a}$ systematically generating knowledge bases over a given signature in $\rho$ RANF that are consistent, pairwise not antecedentwise equivalent and pairwise not equivalent under signature renaming. We also show that, when taking signature renamings and model equivalence into account, every consistent knowledge base is generated, thus providing an excellent base for empirical evaluations and comparisons of conditional knowledge bases. Furthermore, we show that normalizing the set of all knowledge bases over a signature $\Sigma$ to $\rho$ RANF yields exactly the same result as $K B^{\rho r a}(\Sigma)$, highlighting that normal form transformations and systematically generating knowledge bases in normal form are two sides of the same coin.

In summary, the main contributions of this article are:

- Notion of unique renaming normal form $(\rho \mathrm{NF})$ for arbitrary knowledge bases, also across different signatures. 
- Reduced antecedent normal form (RANF) for knowledge bases, taking system P inferences into account.

- Renaming antecedent normal form ( $\rho \mathrm{ANF})$ and renaming reduced antecedent normal form ( $\rho$ RANF), the latter being the most succinct normal form.

- Algorithms for transforming every knowledge base into any of the introduced normal forms, together with corresponding correctness and completeness results.

- Algorithm $K B^{\rho r a}$ for systematically generating all knowledge bases over a given signature in $\rho$ RANF that are consistent, pairwise not antecedentwise equivalent and pairwise not equivalent under signature renaming.

This article is a revised and largely extended version of [19], and it also extends our work presented in [20]. The extensions include in particular the development of a general notion of renaming normal form for arbitrary knowledge bases, the covering of renamings also across different signatures, the provision of algorithms for transforming knowledge bases into any of the normal forms, and the establishment of the formal relationship between normal form transformation on the one hand and the systematic generation of knowledge bases in $\rho$ RANF on the other hand.

The rest of this article is organized in the following way. After recalling the required basics in Section 2, we introduce the renaming normal form in Section 3 and an algorithm to transform arbitrary knowledge bases to renaming normal form in Section 4. The reduced antecedentwise normal form is introduced in Section 5, and the transformation system yielding a knowledge base in RANF is presented in Section 6. The renaming normal form is combined with the ANF and the RANF, respectively, in Section 7. The systematic generation of knowledge bases in $\rho$ RANF and its properties are presented in Section 8. In Section 9, we conclude and point out future work.

\section{Background: conditional logic}

Let $\mathcal{L}(\Sigma)$ be the propositional language over a finite signature $\Sigma$. Unless otherwise stated, $\Sigma$ consists of atoms $a, b, c, \ldots$. We call a signature $\Sigma$ with a linear ordering $\lessdot$ an ordered signature and denote it by $(\Sigma, \lessdot)$. The language may be denoted by $\mathcal{L}$ if the signature is clear from context. The formulas of $\mathcal{L}$ will be denoted by letters $A, B, C, \ldots$. We write $A B$ for $A \wedge B$ and $\bar{A}$ for $\neg A$. We identify the set of all complete conjunctions over $\Sigma$ with the set $\Omega$ of possible worlds over $\mathcal{L}$. For $\omega \in \Omega$ and $A \in \mathcal{L}, \omega \models A$ means that $A$ holds in $\omega$. The set of worlds satisfying $A$ is $\Omega_{A}=\{\omega \mid \omega \models A\}$. Two formulas $A, B$ are equivalent, denoted as $A \equiv B$, if $\Omega_{A}=\Omega_{B}$.

By introducing a new binary operator $\mid$, we obtain the set $(\mathcal{L} \mid \mathcal{L})_{\Sigma}=\{(B \mid A) \mid A, B \in$ $\mathcal{L}(\Sigma)\}$ of conditionals over $\mathcal{L}(\Sigma)$. Again, $\Sigma$ may be omitted. For a conditional $r=(B \mid A)$, $\operatorname{ant}(r)=A$ is the antecedent of $r$, and cons $(r)=B$ is its consequent. The counter conditional of $r=(B \mid A)$ is $\bar{r}=(\bar{B} \mid A)$. As semantics for conditionals, we use ordinal conditional functions $(O C F)$ [21]. An OCF is a function $\kappa: \Omega \rightarrow \mathbb{N}$ expressing degrees of plausibility of possible worlds where a lower degree denotes "less surprising". At least one world must be regarded as being normal; therefore, $\kappa(\omega)=0$ for at least one $\omega \in \Omega$. Each $\kappa$ uniquely extends to a function mapping formulas to $\mathbb{N} \cup\{\infty\}$ given by $\kappa(A)=\min \{\kappa(\omega) \mid \omega \models A\}$ where $\min \emptyset=\infty$. An OCF $\kappa$ accepts a conditional $(B \mid A)$, written $\kappa \models(B \mid A)$, if the verification of the conditional is less surprising than its falsification, i.e., if $\kappa(A B)<\kappa(A \bar{B})$; equivalently, $\kappa \models(B \mid A)$ iff for every $\omega^{\prime} \in \Omega_{A \bar{B}}$ there is $\omega \in \Omega_{A B}$ with $\kappa(\omega)<\kappa\left(\omega^{\prime}\right)$. A conditional $(B \mid A)$ is trivial if it is self-fulfilling $(A \models B)$ or contradictory $(A \models \bar{B})$; a 
set of conditionals is self-fulfilling if every conditional in it is self-fulfilling. A finite set $\mathcal{R} \subseteq(\mathcal{L} \mid \mathcal{L})$ of conditionals is called a knowledge base. An OCF $\kappa$ accepts a knowledge base $\mathcal{R}$ if $\kappa$ accepts all conditionals in $\mathcal{R}$, and $\mathcal{R}$ is consistent if an OCF accepting $\mathcal{R}$ exists [9]. We use $\diamond$ to denote an inconsistent knowledge base. $\operatorname{Mod}(\mathcal{R})$ denotes the set of all OCFs $\kappa$ accepting $\mathcal{R}$. Two knowledge bases $\mathcal{R}, \mathcal{R}^{\prime}$ are model equivalent, denoted by $\mathcal{R} \equiv_{\text {mod }} \mathcal{R}^{\prime}$, if $\operatorname{Mod}(\mathcal{R})=\operatorname{Mod}\left(\mathcal{R}^{\prime}\right)$. Correspondingly, we say that $(B \mid A)$ and $\left(B^{\prime} \mid A^{\prime}\right)$ are equivalent, denoted by $(B \mid A) \equiv\left(B^{\prime} \mid A^{\prime}\right)$, if $A \equiv A^{\prime}$ and $A B \equiv A^{\prime} B^{\prime}$ where $\equiv$ is the propositional equivalence.

System $P$ [7] allows reasoning about conditional knowledge bases. It consists of the six axioms that are displayed in Figure 1. If a conditional $(B \mid A)$ can be derived from a conditional knowledge base $\mathcal{R}$ by applying the rules in system $\mathrm{P}$, we denote this by $A \sim_{\mathcal{R}} B$. It has been shown (see $[3,7,8,22]$ ) that system $\mathrm{P}$ inference coincides with p-entailment [9] where $A$ p-entails $B$ in the context of $\mathcal{R}$ iff all models of $\mathcal{R}$ accept the conditional $(B \mid A)$. Moreover, $A \sim_{\mathcal{R}} B$ holds iff $\mathcal{R} \cup\{(\bar{B} \mid A)\}$ is inconsistent [9].

Two knowledge bases $\mathcal{R}, \mathcal{R}^{\prime}$ are inferentially equivalent, if $A \sim_{\mathcal{R}} B$ holds if and only if $A \sim_{\mathcal{R}^{\prime}} B$ for all formulas $A, B$. Note, that inferential equivalence with respect to system $\mathrm{P}$ and model equivalence coincide.

\section{Renamings and renaming normal form ( $\rho$ NF)}

There are knowledge bases that are identical except for the names of their variables. E.g., the knowledge bases $\mathcal{R}_{1}=\{(a \mid b),(a \mid c)\}$ and $\mathcal{R}_{2}=\{(c \mid b),(c \mid a)\}$ become equal if we swap the names for the variables $a$ and $c$ in one of them.

When analysing the structure of a knowledge base, we are only interested in one of such knowledge bases that are identical except for a signature renaming.

Definition 1 (renaming, $\simeq, \simeq$ mod ) Let $\Sigma, \Sigma^{\prime}$ be signatures. We call a bijective function $\rho: \Sigma \rightarrow \Sigma^{\prime}$ a (signature) renaming. A renaming is lifted canonically to formulas, worlds,

$$
\begin{array}{lc}
(R E) \text { Reflexivity: } & A \sim A \\
(L L E) \text { Left Logical Equivalence: } & \frac{\models A \leftrightarrow B, \quad A \sim C}{B \sim C} \\
(R W) \text { Right Weakening: } & \frac{\models B \rightarrow C, A \sim B}{A \sim C} \\
(A N D) \text { And: } & \frac{A \sim B, A \sim C}{A \sim B \wedge C} \\
(\text { OR) Or: } & \frac{A \sim C, \quad B \sim C}{A \vee B \sim C} \\
(C M) \text { Cautious Monotonicity: } & \frac{A \sim B, \quad A \sim C}{A \wedge B \sim C}
\end{array}
$$

Fig. 1 Axioms of system P (cf. [7]) with $A, B, C \in \mathcal{L}$ 
conditionals, knowledge bases, and sets thereof. Two worlds, formulas, conditionals, knowledge bases, or sets thereof are equivalent under signature renaming, denoted as $X \simeq X^{\prime}$, if there exists a renaming $\rho$ such that $X^{\prime}=\rho(X)$. Two knowledge bases $\mathcal{R}, \mathcal{R}^{\prime}$ over $\Sigma, \Sigma^{\prime}$ are model equivalent up to renamings, denoted $\mathcal{R} \simeq$ mod $\mathcal{R}^{\prime}$, if there is a renaming $\rho: \Sigma^{\prime} \rightarrow \Sigma$ such that $\mathcal{R} \equiv{ }_{\bmod } \rho\left(\mathcal{R}^{\prime}\right)$.

Note that $\mathcal{R} \simeq \mathcal{R}^{\prime}$ implies $\mathcal{R} \simeq{ }_{\text {mod }} \mathcal{R}^{\prime}$, but not vice versa. On the other hand, each of the two conditions $\mathcal{R} \simeq \mathcal{R}^{\prime}$ and $\mathcal{R} \simeq{ }_{\text {mod }} \mathcal{R}^{\prime}$ ensures inferential equivalence up to renamings.

Proposition 1 ( $\sim_{\mathcal{R}}$ and renamings) If $\mathcal{R} \equiv_{\bmod } \rho\left(\mathcal{R}^{\prime}\right)$ for a renaming $\rho$, i.e. $\mathcal{R} \simeq_{\text {mod }} \mathcal{R}^{\prime}$, then $A \sim_{\mathcal{R}^{\prime}} B$ iff $\rho(A) \sim_{\mathcal{R}} \rho(B)$.

Proof Let $\mathcal{R}, \mathcal{R}^{\prime}$ be knowledge bases with $\mathcal{R} \equiv_{\text {mod }} \rho\left(\mathcal{R}^{\prime}\right)$. The semantic of conditional knowledge bases does not depend on which symbols are used in the signature, i.e. $A \sim_{\mathcal{R}^{\prime}} B$ iff $\rho(A) \sim_{\rho\left(\mathcal{R}^{\prime}\right)} \rho(B)$. Two model equivalent knowledge bases have the same models by definition and therefore entail the same conditionals. Hence, $\rho(A) \sim_{\rho\left(\mathcal{R}^{\prime}\right)} \rho(B)$ iff $\rho(A) \sim_{\mathcal{R}} \rho(B)$ because of $\mathcal{R} \equiv_{\text {mod }} \rho\left(\mathcal{R}^{\prime}\right)$.

In order to be able to deal with normal forms of formulas in $\mathcal{L}$ without having to select a specific representation, we assume a function $v$ mapping a propositional formula $A$ to a unique normal form $v(A)$ such that $A \equiv A^{\prime}$ iff $v(A)=v\left(A^{\prime}\right)$.

Definition $2\left(=_{\nu}\right)$ Two propositional formulas $A, A^{\prime}$ are equal under normalization, denoted as $A={ }_{v} A^{\prime}$, if $v(A)=v\left(A^{\prime}\right)$. This equivalence is lifted canonically to sets of formulas. Two conditionals $(B \mid A),\left(B^{\prime} \mid A^{\prime}\right)$ are equal under normalization, if $A={ }_{v} A^{\prime}$ and $B={ }_{v} B^{\prime}$. This equivalence is lifted canonically to sets of conditionals.

Note that the definition of the normalization function implies that for formulas $A, B$ we have $A={ }_{v} B$ iff $A \equiv B$.

Definition $3\left(\simeq_{v}\right)$ Two worlds are equivalent under signature renaming and normalization, denoted as $\omega \simeq_{v} \omega^{\prime}$, if $\omega \simeq \omega^{\prime}$. Two formulas $A, A^{\prime}$ are equivalent under signature renaming and normalization, denoted by $A \simeq_{v} A^{\prime}$, if there exists a renaming $\rho$ such that $\rho(A)={ }_{v} A^{\prime}$.

Two conditionals $(B \mid A),\left(B^{\prime} \mid A^{\prime}\right)$ are equivalent under signature renaming and normalization, denoted by $(B \mid A) \simeq_{v}\left(B^{\prime} \mid A^{\prime}\right)$, if there exists a renaming $\rho$ such that $\rho(A)={ }_{v} A^{\prime}$ and $\rho(B)={ }_{v} B^{\prime}$. As usual, these equivalences are lifted canonically to sets of worlds, formulas, and conditionals, respectively.

Similar as before, $A \simeq_{v} B$ implies $A \simeq_{\text {mod }} B$.

In contrast to renaming, normalization might change the size of a knowledge base, as the following example shows.

Example 3 Let $\mathcal{R}=\{(b \mid a),(b \mid a \vee a)\}$ and $\mathcal{R}^{\prime}=\{(b \mid a)\}$. As both conditionals in $\mathcal{R}$ are equal under normalization, they will be mapped to the same normal form by $v$. Therefore, $\mathcal{R}={ }_{v} \mathcal{R}^{\prime}$ although $|\mathcal{R}| \neq\left|\mathcal{R}^{\prime}\right|$. 
For a set $M, m \in M$, and an equivalence relation $\equiv$ on $M$, the set of equivalence classes induced by $\equiv$ is denoted by $[M]_{/ \equiv}$, and the unique equivalence class containing $m$ is denoted by $[m]_{\equiv}$. It is easy to see that equivalence under signature renaming $\simeq$ and equivalence under signature renaming and normalization $\simeq_{v}$ are equivalence relations. Thus, for instance, the only non-identity renaming from $\Sigma_{a b}=\{a, b\}$ to itself is the function $\rho_{a b}$ with $\rho_{a b}(a)=b$ and $\rho_{a b}(b)=a$. The three equivalence classes of worlds over $\Sigma_{a b}$ are $\left[\Omega_{\Sigma_{a b}}\right]_{/ \simeq}=\{[a b],[a \bar{b}, \bar{a} b],[\bar{a} \bar{b}]\}$, and we have $[(a b \mid a b \vee a \bar{b})]_{\simeq_{v}}=[(a b \mid a b \vee \bar{a} b)]_{\simeq_{v}}$.

Obviously, a signature renaming can map a conditional only to a conditional in the same equivalence class induced by $\simeq$, leading to the following observations.

Proposition 2 Let $\Sigma$ be a signature, let $\mathcal{R}, \mathcal{R}^{\prime} \subseteq(\mathcal{L} \mid \mathcal{L})_{\Sigma}$ be knowledge bases, and let $\rho$ be a renaming on $\Sigma$. Then it holds that:

$$
\begin{array}{cl}
\rho(\mathcal{R})=\mathcal{R}^{\prime} \text { iff } & \text { for every }[r] \simeq \in\left[(\mathcal{L} \mid \mathcal{L})_{\Sigma}\right] / \simeq: \rho(\mathcal{R} \cap[r] \simeq)=\mathcal{R}^{\prime} \cap[r] \simeq \\
\mathcal{R} \simeq \mathcal{R}^{\prime} \text { implies } & \text { for every }[r]_{\simeq} \in\left[(\mathcal{L} \mid \mathcal{L})_{\Sigma}\right] / \simeq:|\mathcal{R} \cap[r] \simeq|=\left|\mathcal{R}^{\prime} \cap[r] \simeq\right|
\end{array}
$$

Proof A conditional $r$ can only be renamed within the equivalence class $[r] \simeq$. Therefore, if $\mathcal{R}^{\prime}=\rho(\mathcal{R})$, then $\mathcal{R}^{\prime} \cap[r]_{\simeq}=\rho(\mathcal{R}) \cap[r]_{\simeq}=\rho\left(\mathcal{R} \cap[r]_{\simeq}\right)$, implying the left-to-right direction in (1). For the other direction, if $\rho\left(\mathcal{R} \cap[r]_{\simeq}\right)=\mathcal{R}^{\prime} \cap[r]_{\simeq}$ for every $[r]_{\simeq} \in$

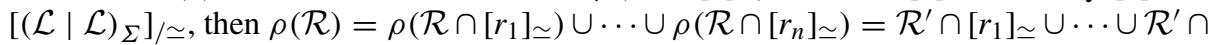
$\left[r_{n}\right]_{\simeq}=\mathcal{R}^{\prime}$ with $\left[(\mathcal{L} \mid \mathcal{L})_{\Sigma}\right]_{/ \simeq}=\left\{\left[r_{1}\right]_{\simeq}, \ldots,\left[r_{n}\right]_{\simeq}\right\}$. Hence, the first observation holds.

The second observation (2) is an immediate consequence of (1).

Thus, the first observation (1) states that a renaming $\rho$ maps $\mathcal{R}$ to $\mathcal{R}^{\prime}$ if and only if for every equivalence class $[r] \simeq$ with respect to $\simeq$, it maps the conditionals in $\mathcal{R}$ from $[r] \simeq$ to the conditionals in $\mathcal{R}^{\prime}$ from $[r] \simeq$. Later on, we will use this observation to check whether two knowledge bases $\mathcal{R}, \mathcal{R}^{\prime}$ are equivalent under renaming by checking if $\rho\left(\mathcal{R} \cap[r]_{\simeq}\right)=$ $\mathcal{R}^{\prime} \cap[r]_{\simeq}$ for every equivalence class $[r] \simeq \in\left[(\mathcal{L} \mid \mathcal{L})_{\Sigma}\right]_{/ \simeq \text {. }}$

To define a normal form that abstracts from renamings, we have to select one element from each equivalence class $[r]_{\simeq}$. For this, we use an ordering on $(\mathcal{L} \mid \mathcal{L})_{\Sigma}$. For our approach, it is not important which ordering exactly we use as long as it fulfils the following properties.

Definition 4 (admissible $\preccurlyeq$ on $\left.(\mathcal{L} \mid \mathcal{L})_{\Sigma}\right)$ A total preorder $\preccurlyeq$ on $(\mathcal{L} \mid \mathcal{L})_{\Sigma}$ is admissible if it fulfils the following conditions:

1. For any two conditionals $c_{1}, c_{2}$ with $c_{1} \neq v c_{2}$ we have either $c_{1} \preccurlyeq c_{2}$ or $c_{2} \preccurlyeq c_{1}$ but not both.

2. For any two conditionals $c_{1}, c_{2}$ with $c_{1}={ }_{\nu} c_{2}$ we have both $c_{1} \preccurlyeq c_{2}$ and $c_{2} \preccurlyeq c_{1}$.

3. For any two equivalence classes $\left[c_{1}\right]_{\simeq_{v}},\left[c_{2}\right]_{\simeq_{v}}$ with $\left[c_{1}\right] \simeq_{v} \neq\left[c_{2}\right] \simeq_{v}$ and $c_{1} \preccurlyeq c_{2}$ it holds that for every $c_{1}^{\prime} \in\left[c_{1}\right]_{\simeq_{v}}, c_{2}^{\prime} \in\left[c_{2}\right]_{\simeq_{v}}$ we have $c_{1}^{\prime} \preccurlyeq c_{2}^{\prime}$.

For developing such an order, we will represent each formula $A \in \mathcal{L}$ uniquely by its set $\Omega_{A}$ of satisfying worlds. Furthermore, we use the following notation. For an ordering relation $\leqslant$ on a set $M$, its lexicographic extension to strings over $M$ is denoted by $\leqslant$ lex. For ordered sets $S, S^{\prime} \subseteq M$ with $S=\left\{e_{1}, \ldots, e_{n}\right\}$ and $S^{\prime}=\left\{e_{1}^{\prime}, \ldots, e_{n^{\prime}}^{\prime}\right\}$ where $e_{i} \leqslant e_{i+1}$ for $i \in\{1, \ldots, n-1\}$ and $e_{j}^{\prime} \leqslant e_{j+1}^{\prime}$ for $j \in\left\{1, \ldots, n^{\prime}-1\right\}$, its extension $\leqslant$ set to sets is:

$$
\begin{aligned}
& S \leqslant_{\text {set }} S^{\prime} \text { iff } n<n^{\prime}, \\
& \text { or } n=n^{\prime} \text { and } e_{1} \ldots e_{n} \leqslant_{l e x} e_{1}^{\prime} \ldots e_{n^{\prime}}^{\prime}
\end{aligned}
$$


For an ordered signature $(\Sigma, \lessdot)$ with linear order $\lessdot$, let $\llbracket \omega \rrbracket_{\lessdot}$ denote the usual interpretation of a world $\omega$ as a binary number; e.g., for $\Sigma_{a b}$ with $a \lessdot b, \llbracket a b \rrbracket_{\lessdot}=3$, $\llbracket a \bar{b} \rrbracket_{\lessdot}=2$, $\llbracket \bar{a} b \rrbracket_{\lessdot}=1$, and $\llbracket \bar{a} \bar{b} \rrbracket \rrbracket_{\lessdot}=0$.

As usual for any ordering $\preccurlyeq$, we denote the strict variant with $\prec$, i.e., $c_{1} \prec c_{2}$ iff $c_{1} \preccurlyeq c_{2}$ and $c_{2} \npreceq c_{1}$.

Definition 5 (induced ordering on formulas and conditionals $\stackrel{w}{\leftarrow}, \stackrel{c}{\lessgtr}$ ) Let $\Sigma$ be a signature with linear ordering $\lessdot$. The orderings induced by $\lessdot$ on worlds $\omega, \omega^{\prime}$ and conditionals $(B \mid A),\left(B^{\prime} \mid A^{\prime}\right)$ over $\Sigma$ are given by:

$$
\begin{aligned}
\omega \stackrel{w}{\omega} \omega^{\prime} & \text { iff } \llbracket \omega \rrbracket_{\lessdot} \geqslant \llbracket \omega^{\prime} \rrbracket_{\lessdot} \\
(B \mid A) \stackrel{c}{\leftarrow}\left(B^{\prime} \mid A^{\prime}\right) & \text { iff } \Omega_{A} \stackrel{w}{\leftarrow_{s e t}} \Omega_{A^{\prime}}, \\
& \text { or } \Omega_{A}=\Omega_{A^{\prime}} \text { and } \Omega_{B} \stackrel{w}{\aleph_{s e t}} \Omega_{B^{\prime}}
\end{aligned}
$$

In order to ease our notation, we will omit the upper symbol in $\stackrel{w}{\lessgtr}$ and $\stackrel{c}{\lessgtr}$, and write just $\leqslant$ instead, and analogously $\lessdot$ for the strict variants. For instance, for $\Sigma_{a b}$ with $a \lessdot b$ we have $a b \lessdot a \bar{b} \lessdot \bar{a} b \lessdot \bar{a} \bar{b}$ for worlds, and $(a b \mid a b \vee a \bar{b}) \lessdot(a b \mid a b \vee \bar{a} \bar{b})$ and $(a b \vee \bar{a} \bar{b} \mid a b \vee$ $a \bar{b} \vee \bar{a} \bar{b}) \lessdot(\bar{a} \bar{b} \mid a b \vee a \bar{b} \vee \bar{a} b \vee \bar{a} \bar{b})$ for conditionals.

Definition 6 (canonical ordering $\preccurlyeq$ ) Given a signature $\Sigma$ with linear ordering $\lessdot$, let $\left[(\mathcal{L} \mid \mathcal{L})_{\Sigma}\right]_{/ \simeq_{v}}=\left\{\left[r_{1}\right]_{\simeq_{v}}, \ldots,\left[r_{m}\right]_{\simeq_{v}}\right\}$ be the equivalence classes of $(\mathcal{L} \mid \mathcal{L})_{\Sigma}$ induced by renamings and normalization such that for each $i \in\{1, \ldots, m\}$, the conditional $r_{i}$ is a minimal element in $\left[r_{i}\right]_{\simeq_{v}}$ with respect to $\leqslant$, and $r_{1} \lessdot \ldots \lessdot r_{m}$. The minimal conditionals in each $\left[r_{i}\right]_{\simeq_{v}}$ with respect to $\lessdot$ are called the canonical conditionals over $\Sigma$. The canonical ordering on $(\mathcal{L} \mid \mathcal{L})_{\Sigma}$ induced by $\preccurlyeq$, denoted by $\preccurlyeq$, is given by the schema

$$
\left[r_{1}\right]_{\simeq_{v}} \prec\left[r_{2}\right]_{\simeq_{v}} \prec \ldots \prec\left[r_{m}\right]_{\simeq_{v}}
$$

where conditionals $r, r^{\prime} \in\left[r_{i}\right]_{\simeq_{v}}$ in each equivalence class $\left[r_{i}\right]_{\simeq_{v}}$, for $i \in\{1, \ldots, m\}$, are ordered by $r \preccurlyeq r^{\prime}$ iff $r \leqslant r^{\prime}$.

For example, we have $(a b \mid a b \vee a \bar{b}) \prec(a b \mid a b \vee \bar{a} b)$ because

$$
[(a b \mid a b \vee a \bar{b})]_{\simeq_{v}}=[(a b \mid a b \vee \bar{a} b)]_{\simeq_{v}} \quad \text { and } \quad(a b \mid a b \vee a \bar{b}) \lessdot(a b \mid a b \vee \bar{a} b) .
$$

Furthermore, we have $(a b \mid a b \vee \bar{a} b) \prec(a \bar{b} \mid a b \vee a \bar{b})$ because

$$
[(a b \mid a b \vee \bar{a} b)]_{\simeq_{v}} \neq[(a \bar{b} \mid a b \vee a \bar{b})]_{\simeq_{v}}
$$

and

$$
\min _{\preccurlyeq}\left([(a b \mid a b \vee \bar{a} b)]_{\simeq_{v}}\right)=(a b \mid a b \vee a \bar{b}) \lessdot(a \bar{b} \mid a b \vee a \bar{b})=\min _{\preccurlyeq}((a \bar{b} \mid a b \vee a \bar{b}))
$$

even though $(a \bar{b} \mid a b \vee a \bar{b}) \lessdot(a b \mid a b \vee \bar{a} b)$.

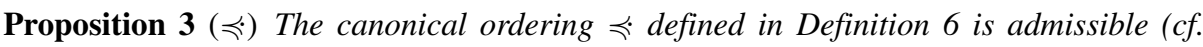
Definition 4).

Proof We show that $\leqslant$ fulfils conditions (1.) and (2.) for admissibility first. Consider $c_{1}=$ $\left(B_{1} \mid A_{1}\right), c_{2}=\left(B_{2} \mid A_{2}\right)$. If $c_{1}={ }_{v} c_{2}$ then $\Omega_{A_{1}}=\Omega_{A_{2}}$ and $\Omega_{B_{1}}=\Omega_{B_{2}}$. Hence, both $c_{1} \stackrel{c}{\leqslant} c_{2}$ 
and $c_{2} \stackrel{c}{\stackrel{c}{\leftarrow}} c_{1}$ hold. If $c_{1} \neq \neq_{v} c_{2}$ then either $\Omega_{A_{1}} \neq \Omega_{A_{2}}$ or $\Omega_{B_{1}} \neq \Omega_{B_{2}}$. Hence, either $c_{1} \stackrel{c}{\leftarrow} c_{2}$ or $c_{2} \stackrel{c}{\leftarrow} c_{1}$ holds. Because the ordering $\preccurlyeq$ compared to $\leqslant$ only rearranges the ordering of the equivalence classes with respect to $=_{\nu}$, the conditions (1.) and (2.) of admissibility can be transferred from $\preccurlyeq$ to $\preccurlyeq$. The schema of $\preccurlyeq$ given in Definition 6 shows that $\preccurlyeq$ fulfils condition (3.) of admissibility.

In the following, we will abbreviate $\mathcal{R} \preccurlyeq$ set $\mathcal{R}^{\prime}$ simply by $\mathcal{R} \preccurlyeq \mathcal{R}^{\prime}$ for knowledge bases $\mathcal{R}, \mathcal{R}^{\prime}$, and analogously for the strict version $\prec_{\text {set }}$. Using these notations, we can introduce the notion of renaming normal form for knowledge bases containing any conditionals over $\Sigma$.

Definition 7 (renaming normal form, $\rho$ NF) A knowledge base $\mathcal{R}$ over an ordered signature $(\Sigma, \lessdot)$ is in renaming normal form $(\rho \mathrm{NF})$ if for every knowledge base $\mathcal{R}^{\prime}$ over $\Sigma$ with $\mathcal{R} \simeq \mathcal{R}^{\prime}$ we have $\mathcal{R} \preccurlyeq \mathcal{R}^{\prime}$.

Note that while we use the renaming equivalence $\simeq$ in Definition 7 , the next proposition shows that it does not make any difference if we use the renaming equivalence under normalization $\simeq_{v}$ instead.

Proposition 4 A knowledge base $\mathcal{R}$ over an ordered signature $(\Sigma, \lessdot)$ is in $\rho$ NF iff for every knowledge base $\mathcal{R}^{\prime}$ over $\Sigma$ with $\mathcal{R} \simeq_{v} \mathcal{R}^{\prime}$ we have $\mathcal{R} \preccurlyeq \mathcal{R}^{\prime}$.

Proof We prove this proposition by showing both implications of the "iff".

$\Rightarrow$ : Let $\mathcal{R}$ be a knowledge base in $\rho \mathrm{NF}$ and $\mathcal{R} \simeq_{v} \mathcal{R}^{\prime}$. Let $\rho$ be the renaming such that $\rho(\mathcal{R})={ }_{v} \mathcal{R}^{\prime}$. Because $\mathcal{R}$ is in $\rho \mathrm{NF}$, we have $\mathcal{R} \preccurlyeq \rho(\mathcal{R}) \preccurlyeq \mathcal{R}^{\prime}$ (cf. condition (2.) of admissibility in Definition 4$)$.

$\Leftarrow: \quad$ Let $\mathcal{R}$ be a knowledge base such that $\mathcal{R} \preccurlyeq \mathcal{R}^{\prime}$ for all $\mathcal{R}^{\prime}$ such that $\mathcal{R} \simeq_{v} \mathcal{R}^{\prime}$. This implies that $\mathcal{R} \preccurlyeq \mathcal{R}^{\prime}$ for all $\mathcal{R}^{\prime}$ such that $\mathcal{R} \simeq \mathcal{R}^{\prime}$ and therefore that $\mathcal{R}$ is in $\rho \mathrm{NF}$.

For every knowledge base, a corresponding knowledge base in $\rho$ NF exists. More precisely, we have:

Proposition 5 ( $\rho \mathrm{NF})$ For every consistent conditional knowledge base $\mathcal{R}$ over an ordered signature $(\Sigma, \lessdot)$ there is a knowledge base $\mathcal{R}^{\prime}$ in $\rho N F$ over $\Sigma$ such that $R \simeq R^{\prime}$.

If two knowledge bases $\mathcal{R}^{\prime}, \mathcal{R}^{\prime \prime}$ over $\Sigma$ are both in $\rho N F$ and $\mathcal{R} \simeq \mathcal{R}^{\prime}$ and $\mathcal{R} \simeq \mathcal{R}^{\prime \prime}$, then $\mathcal{R}^{\prime}={ }_{v} \mathcal{R}^{\prime \prime}$.

Proof Consider the set $\left\{\mathcal{R}^{\prime} \subseteq(\mathcal{L} \mid \mathcal{L})_{\Sigma} \mid R \simeq R^{\prime}\right\}$ of all knowledge bases over $\Sigma$ that are renaming equivalent to $\mathcal{R}$. Select a minimal element with respect to $\preccurlyeq$ from this set. This element is in $\rho$ NF. With condition 1 of the definition of admissibility it follows that $\mathcal{R}^{\prime}={ }_{v} \mathcal{R}^{\prime \prime}$.

In the strict sense, for every knowledge base $\mathcal{R}$, there is a set $S=\left\{\mathcal{R}^{\prime} \subseteq(\mathcal{L} \mid \mathcal{L})_{\Sigma} \mid \mathcal{R} \simeq\right.$ $\mathcal{R}^{\prime}$ and $\mathcal{R}^{\prime}$ is in $\left.\rho \mathrm{NF}\right\}$ that is not necessarily a singleton. But Proposition 5 states that all elements of $S$ are equivalent under propositional normalization. Therefore, in cases where it does not matter which element from $S$ is selected, we will denote some arbitrary element from $S$ as $\rho \mathrm{NF}(\mathcal{R})$ and call it "the" $\rho \mathrm{NF}$ of $\mathcal{R}$. 
An interesting property of the $\rho \mathrm{NF}$ is that it respects equivalence under (propositional) normalization. Furthermore, two knowledge bases that are equivalent under renaming share the same $\rho \mathrm{NF}$.

Proposition 6 Let $\mathcal{R}, \mathcal{R}^{\prime}$ be two consistent conditional knowledge bases over the same ordered signature.

1. $\mathcal{R}={ }_{v} \mathcal{R}^{\prime}$ implies $\rho N F(\mathcal{R})={ }_{v} \rho N F\left(\mathcal{R}^{\prime}\right)$.

2. $\mathcal{R} \simeq \mathcal{R}^{\prime}$ implies $\rho N F(\mathcal{R})={ }_{\nu} \rho N F\left(\mathcal{R}^{\prime}\right)$.

3. $\mathcal{R} \simeq{ }_{v} \mathcal{R}^{\prime}$ iff $\rho N F(\mathcal{R})={ }_{v} \rho N F\left(\mathcal{R}^{\prime}\right)$.

\section{Proof}

(1.) $\quad$ Assume $\rho \mathrm{NF}(\mathcal{R}) \neq{ }_{v} \rho \mathrm{NF}\left(\mathcal{R}^{\prime}\right)$. W.l.o.g. we assume $\rho \mathrm{NF}(\mathcal{R}) \prec \rho \mathrm{NF}\left(\mathcal{R}^{\prime}\right)$. Let $\rho$ be the renaming that transforms $\mathcal{R}$ to $\rho \mathrm{NF}(\mathcal{R})={ }_{v} \rho(\mathcal{R})$. We have $\rho\left(\mathcal{R}^{\prime}\right)={ }_{v} \rho(\mathcal{R})$ and therefore $\rho\left(\mathcal{R}^{\prime}\right) \preccurlyeq \rho \mathrm{NF}(\mathcal{R}) \prec \rho \mathrm{NF}\left(\mathcal{R}^{\prime}\right)$. This is a contradiction.

(3.) We will show both implications of the "iff".

$$
\Leftarrow: \quad \mathcal{R} \simeq{ }_{v} \rho \mathrm{NF}(\mathcal{R})={ }_{v} \rho \mathrm{NF}\left(\mathcal{R}^{\prime}\right) \simeq_{v} \mathcal{R}^{\prime}
$$

$\Rightarrow: \quad \mathcal{R} \simeq_{v} \mathcal{R}^{\prime}$ implies that there is a renaming $\rho$ such that $\rho(\mathcal{R})={ }_{v} \mathcal{R}^{\prime}$. Let $\rho^{\prime}$ be the renaming that maps $\mathcal{R}^{\prime}$ to $\rho \mathrm{NF}\left(\mathcal{R}^{\prime}\right)$. Then $\rho^{\prime} \circ \rho$ maps $\mathcal{R}$ to $\rho \mathrm{NF}\left(\mathcal{R}^{\prime}\right)$ which is in $\rho \mathrm{NF}$. With Proposition 5 it follows that $\rho \operatorname{NF}(\mathcal{R})={ }_{v} \rho^{\prime} \circ \rho(\mathcal{R})=\rho \mathrm{NF}\left(\mathcal{R}^{\prime}\right)$.

(2.) This is an implication of (3.).

Removing the $\preccurlyeq$-maximal element form a knowledge base that is in $\rho$ NF yields a knowledge base that is also in $\rho \mathrm{NF}$.

Proposition 7 Let $\mathcal{R}$ be a knowledge base in $\rho N F$ and $r$ be a maximal conditional in $\mathcal{R}$ with respect to $\preccurlyeq$. Then $\mathcal{R} \backslash\{r\}$ is in $\rho N F$ as well.

Proof Assume there be a renaming $\rho$, such that $\rho(\mathcal{R} \backslash\{r\}) \prec \mathcal{R} \backslash\{r\}$. Then according to (3), $\rho(\mathcal{R}) \prec \mathcal{R}$ because $r$ is a maximum of $\mathcal{R}$. This is a contradiction to the assumption that $\mathcal{R}$ is in $\rho \mathrm{NF}$.

This property will be exploited in Section 8 for systematically generating knowledge bases in $\rho$ NF.

So far, the renamings taken into account are mappings from a signature to itself. We can also consider renamings across different signatures.

Proposition 8 For every consistent conditional knowledge base $\mathcal{R}$ over $(\mathcal{L} \mid \mathcal{L})_{\Sigma}$ and every ordered signature $\left(\Sigma^{\prime}, \lessdot\right)$ such that $|\Sigma|=\left|\Sigma^{\prime}\right|$ there is a knowledge base $\mathcal{R}^{\prime} \subseteq(\mathcal{L} \mid \mathcal{L})_{\Sigma^{\prime}}$ in $\rho N F$ such that $\mathcal{R} \simeq \mathcal{R}^{\prime}$.

If two knowledge bases $\mathcal{R}^{\prime}, \mathcal{R}^{\prime \prime} \in(\mathcal{L} \mid \mathcal{L})_{\Sigma^{\prime}}$ are both in $\rho N F$ and $\mathcal{R} \simeq \mathcal{R}^{\prime}$ and $\mathcal{R} \simeq \mathcal{R}^{\prime \prime}$ then $\mathcal{R}^{\prime}={ }_{v} \mathcal{R}^{\prime \prime}$.

Proof As $|\Sigma|=\left|\Sigma^{\prime}\right|$ there is a renaming $\rho: \Sigma \rightarrow \Sigma^{\prime}$ that maps $\mathcal{R}$ to a knowledge base $\mathcal{R}^{\prime \prime \prime}$ over $\Sigma^{\prime}$. With Proposition 5, it follows that there exists a knowledge base $\mathcal{R}^{\prime}$ in $\rho \mathrm{NF}$ such that $\mathcal{R}^{\prime \prime \prime} \simeq \mathcal{R}^{\prime}$. Therefore, $\mathcal{R} \simeq \mathcal{R}^{\prime \prime \prime} \simeq \mathcal{R}^{\prime}$. 
From $\mathcal{R}^{\prime} \simeq \mathcal{R} \simeq \mathcal{R}^{\prime \prime}$, it follows with Proposition 6 that $\mathcal{R}^{\prime}={ }_{v} \mathcal{R}^{\prime \prime}$.

Again, in general, for every knowledge base $\mathcal{R}$ there is a, possibly non-singelton, set $S=\left\{\mathcal{R}^{\prime} \in(\mathcal{L} \mid \mathcal{L})_{\Sigma^{\prime}} \mid \mathcal{R} \simeq \mathcal{R}^{\prime}\right.$ and $\mathcal{R}^{\prime}$ is in $\left.\rho \mathrm{NF}\right\}$ of renaming equivalent knowledge bases in $\rho \mathrm{NF}$. Proposition 8 allows us to write $\rho \mathrm{NF}_{\Sigma^{\prime}}(\mathcal{R})$ to denote an arbitrary element from $S$ if it does not make a difference which element is selected (cf. $\rho \mathrm{NF}(\mathcal{R})$ ).

Proposition 6 can be transferred to situations with multiple signatures.

Proposition 9 Let $\mathcal{R}^{\prime} \subseteq(\mathcal{L} \mid \mathcal{L})_{\Sigma^{\prime}}, \mathcal{R}^{\prime \prime} \subseteq(\mathcal{L} \mid \mathcal{L})_{\Sigma^{\prime \prime}}$ be two consistent knowledge bases and $(\Sigma, \lessdot)$ be an ordered signature.

Then $\mathcal{R}^{\prime} \simeq_{v} \mathcal{R}^{\prime \prime}$ iff $\rho N F_{\Sigma}\left(\mathcal{R}^{\prime}\right)={ }_{v} \rho N F_{\Sigma}\left(\mathcal{R}^{\prime \prime}\right)$.

Proof We will show both implications of the "iff".

$\Rightarrow$ : Let $\mathcal{R}^{\prime} \simeq_{v} \mathcal{R}^{\prime \prime}$. Then there is a renaming $\rho$ such that $\rho\left(\mathcal{R}^{\prime}\right)={ }_{v} \mathcal{R}^{\prime \prime}$. Therefore, $\rho \mathrm{NF}_{\Sigma}\left(\mathcal{R}^{\prime}\right)={ }_{\nu} \rho \mathrm{NF}_{\Sigma}\left(\rho\left(\mathcal{R}^{\prime}\right)\right)={ }_{\nu} \rho \mathrm{NF}_{\Sigma}\left(\mathcal{R}^{\prime \prime}\right)$.

$\Leftarrow$ : Let $\rho \mathrm{NF}_{\Sigma}\left(\mathcal{R}^{\prime}\right)={ }_{\nu} \rho \mathrm{NF}_{\Sigma}\left(\mathcal{R}^{\prime \prime}\right)$. Then there are renamings $\rho^{\prime}, \rho^{\prime \prime}$ such that $\rho^{\prime}\left(\mathcal{R}^{\prime}\right)={ }_{v} \rho \mathrm{NF}_{\Sigma}\left(\mathcal{R}^{\prime}\right)$ and $\rho^{\prime \prime}\left(\mathcal{R}^{\prime \prime}\right)={ }_{v} \rho \mathrm{NF}_{\Sigma}\left(\mathcal{R}^{\prime \prime}\right)$. Therefore, $\rho^{\prime}\left(\mathcal{R}^{\prime}\right)={ }_{v} \rho^{\prime \prime}\left(\mathcal{R}^{\prime \prime}\right)$. As renaming does not affect model equivalence, we have $\rho^{\prime \prime-1}\left(\rho^{\prime}\left(\mathcal{R}^{\prime}\right)\right)={ }_{v} \mathcal{R}^{\prime \prime}$. Because $\rho^{\prime \prime-1} \circ \rho^{\prime}$ is a renaming from $\Sigma^{\prime}$ to $\Sigma^{\prime \prime}$, we have $\mathcal{R}^{\prime} \simeq_{v} \mathcal{R}^{\prime \prime}$.

Thus, for comparing knowledge bases over different signatures with respect to equivalence under renaming and normalization, we can compare their renaming normal forms with respect to some ordered signature $\Sigma$.

\section{Converting Knowledge Bases to $\rho$ NF}

In this section, we will introduce an algorithm transforming an arbitrary conditional knowledge base over an ordered signature into $\rho \mathrm{NF}$.

Let $\left[(\mathcal{L} \mid \mathcal{L})_{\Sigma}\right]_{/ \simeq}=\left\{C_{1}, \ldots, C_{n}\right\}$ be the set of equivalence classes of all conditionals

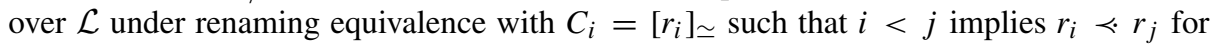
$i, j \in\{1, \ldots, n\}$. The algorithm exploits two main observations:

(O1) For each equivalence class $C_{i}$, every conditional $c \in C_{i}$ is mapped to $C_{i}$ by all renamings over $\Sigma$ (see Proposition 2).

(O2) For any two knowledge bases $\mathcal{R}, \mathcal{R}^{\prime}$ over $\Sigma$ and $1 \leqslant i \leqslant n$ the following implication holds:

$$
\mathcal{R} \cap\left(C_{1} \cup \cdots \cup C_{i}\right) \prec \mathcal{R}^{\prime} \cap\left(C_{1} \cup \cdots \cup C_{i}\right) \text { implies that } \mathcal{R} \prec \mathcal{R}^{\prime} .
$$

The first observation (O1) follows from the definition of the equivalence classes $C_{1}, \ldots, C_{n}$. The second observation (O2) follows from condition 3 of the admissibility of $\preccurlyeq$ (Definition 4).

In combination, these observations imply:

(O3) For any knowledge base $\mathcal{R}$ over $\Sigma$ and $1 \leqslant i \leqslant n$ the following implication holds:

If a renaming $\rho$ maps $\mathcal{R}$ to a knowledge base in $\rho \mathrm{NF}$, then it also maps $\mathcal{R} \cap$ $\left(C_{1} \cup \cdots \cup C_{i}\right)$ to a knowledge base in $\rho$ NF.

Algorithm $\Theta^{\rho}$ (Algorithm 1) can be seen as an implementation of $\rho \mathrm{NF}(\mathcal{R})$. It uses $\operatorname{Perm}(\Sigma)$ to denote the set of all renamings from $\Sigma$ to $\Sigma$. 


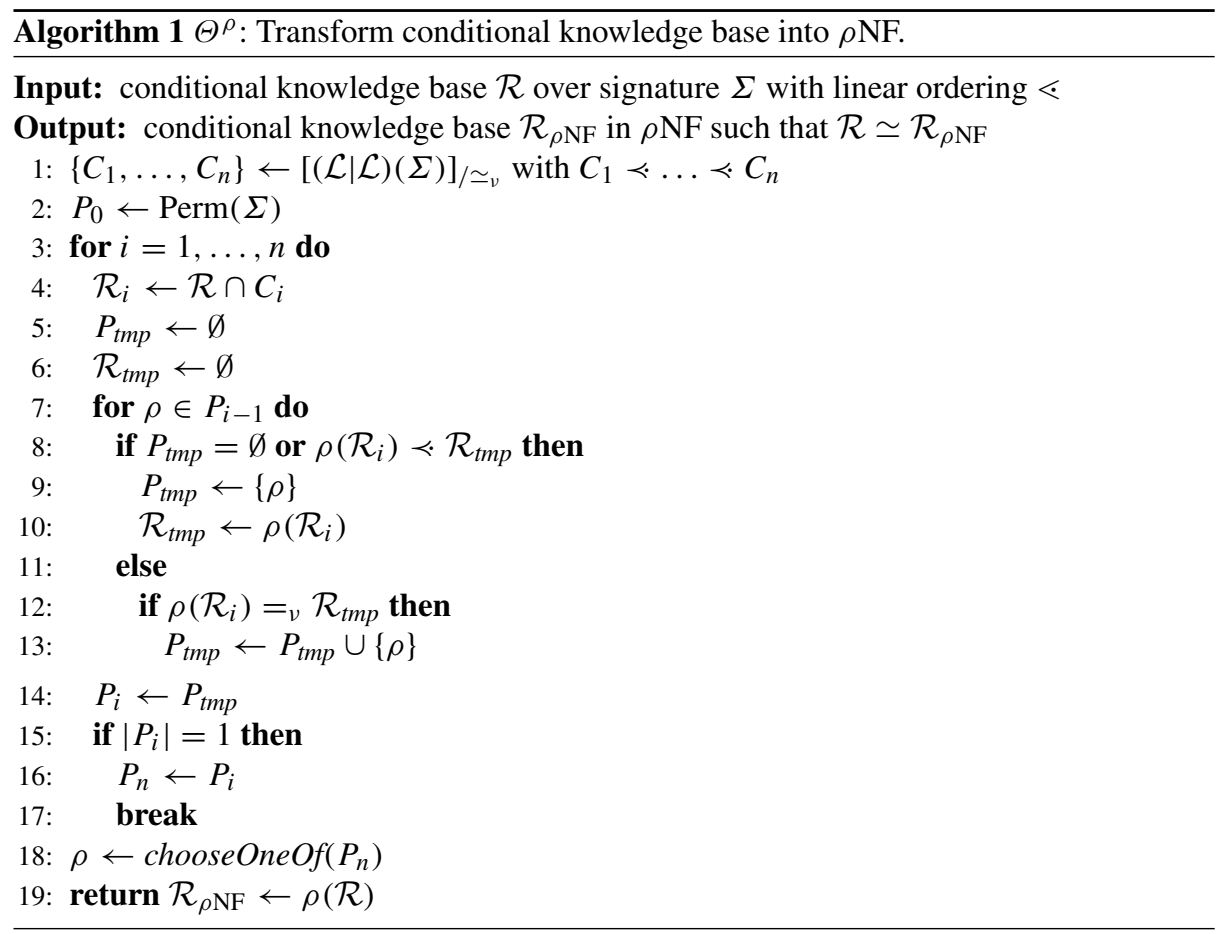

The algorithm $\Theta^{\rho}$ looks for a renaming that transforms a knowledge base $\mathcal{R}$ to $\rho$ NF in Lines 2 to 18. To do so, it starts with the set of all renamings (cf. Line 2) and filters them in each iteration of the for loop starting in Line 3. Using observation (O3), in the $i$-th iteration we keep only the renamings that map $\mathcal{R} \cap\left(C_{1} \cup \cdots \cup C_{i}\right)$ to its $\rho \mathrm{NF}$. After the loop, only renamings that map $\mathcal{R}$ to its $\rho \mathrm{NF}$ remain in $P_{n}$. The algorithm chooses one of these renamings (cf. Line 18) and applies it to $\mathcal{R}$ (cf. Line 19).

Inside the outer loop in Lines 3 to 17, we employ another optimization in Lines 5 to 14 . We know that at the beginning of the $i$-th iteration, all $\rho \in P_{i-1} \operatorname{map} \mathcal{R} \cap\left(C_{1} \cup \cdots \cup C_{i-1}\right)$ to the same knowledge base under normalisation (which is in $\rho \mathrm{NF}$ ). Together with the second observation $(\mathrm{O} 2)$, it follows that:

$$
\begin{aligned}
& \rho_{1}\left(\mathcal{R} \cap\left(C_{1} \cup \cdots \cup C_{i}\right)\right) \preccurlyeq \rho_{2}\left(\mathcal{R} \cap\left(C_{1} \cup \cdots \cup C_{i}\right)\right) \\
& \text { iff } \rho_{1}\left(\mathcal{R} \cap C_{i}\right) \preccurlyeq \rho_{2}\left(\mathcal{R} \cap C_{i}\right)
\end{aligned}
$$

Therefore, it suffices to select those renamings that minimize $\mathcal{R} \cap C_{i}$. This is what happens in Lines 5 to 14.

The check in Lines 15 to 17 avoids unnecessary iterations as there are no non-minimal elements in a singleton.

Formalizing these observations about $\Theta^{\rho}$ yields:

Proposition $10\left(\Theta^{\rho}\right)$ Let $\mathcal{R}$ be a knowledge base.

1. (termination) $\quad \Theta^{\rho}$ terminates on input $\mathcal{R}$.

2. $\left(=_{v}\right) \quad$ If $\rho_{1}, \rho_{2}$ are different choices in Line 18 of executing $\Theta^{\rho}(\mathcal{R})$, then $\rho_{1}(\mathcal{R})={ }_{\nu} \rho_{2}(\mathcal{R})$. 

3. $(\rho N F)$
$\Theta^{\rho}(\mathcal{R})$ is in $\rho N F$.
4. $(\simeq)$
$\mathcal{R} \simeq \Theta^{\rho}(\mathcal{R})$.

In the remainder of this section, we will illustrate two approaches how the algorithm $\Theta^{\rho}$ can be used to check whether two knowledge bases are renaming equivalent.

The first approach transforms two knowledge bases into $\rho \mathrm{NF}$ over their corresponding signatures. Afterwards the knowledge bases are compared. This approach makes use of the following proposition.

Proposition 11 Let $\Sigma_{1}=\left\{a_{1}, \ldots, a_{k}\right\}, \Sigma_{2}=\left\{b_{1}, \ldots, b_{k}\right\}$ be two ordered signatures with the same size with $a_{1} \lessdot_{1} a_{2} \lessdot_{1} \ldots \lessdot_{1} a_{k}$ and $b_{1} \lessdot_{2} b_{2} \lessdot_{2} \ldots \lessdot_{2} b_{k}$. Define $t: \Sigma_{1} \rightarrow$ $\Sigma_{2}, a_{i} \mapsto b_{i}$ for $i=1, \ldots, k$. Let $\mathcal{R}_{1}$ be a knowledge base over $\Sigma_{1}$ and $\mathcal{R}_{2}$ be a knowledge base over $\Sigma_{2}$. Then $\mathcal{R}_{1} \simeq_{v} \mathcal{R}_{2}$ iff $t\left(\Theta^{\rho}\left(\mathcal{R}_{1}\right)\right)={ }_{v} \Theta^{\rho}\left(\mathcal{R}_{2}\right)$.

Proof We will prove both implications of the "iff":

$\Leftarrow$ : We have $\mathcal{R}_{1} \simeq_{v} \Theta^{\rho}\left(\mathcal{R}_{1}\right) \simeq_{v} \Theta^{\rho}\left(\mathcal{R}_{2}\right) \simeq_{v} \mathcal{R}_{2}$ as $t$ is a signature renaming.

$\Rightarrow$ : Assume that $\mathcal{R}_{1} \simeq{ }_{v} \mathcal{R}_{2}$ but $t\left(\Theta^{\rho}\left(\mathcal{R}_{1}\right)\right) \neq{ }_{v} \Theta^{\rho}\left(\mathcal{R}_{2}\right)$. Therefore, we have either $t\left(\Theta^{\rho}\left(\mathcal{R}_{1}\right)\right) \prec \Theta^{\rho}\left(\mathcal{R}_{2}\right)$ or $\Theta^{\rho}\left(\mathcal{R}_{2}\right) \prec t\left(\Theta^{\rho}\left(\mathcal{R}_{1}\right)\right.$ ) (cf. admissibility of $\preccurlyeq$, condition 3 in Definition 4). The first case is impossible because $t\left(\Theta^{\rho}\left(\mathcal{R}_{2}\right)\right)$ is in $\rho \mathrm{NF}$. The ordering of the elements in $\Sigma_{1}$ and $\Sigma_{2}$ together with the definition of $t$ implies that $\mathcal{R} \prec \mathcal{R}^{\prime}$ iff $t(\mathcal{R}) \prec$ $t\left(\mathcal{R}^{\prime}\right)$ for all knowledge bases $\mathcal{R}, \mathcal{R}^{\prime}$. The second case therefore implies $t^{-1}\left(\Theta^{\rho}\left(\mathcal{R}_{2}\right)\right) \prec$ $\Theta^{\rho}\left(\mathcal{R}_{1}\right)$. This is a contradiction, as $\Theta^{\rho}\left(\mathcal{R}_{1}\right)$ is in $\rho \mathrm{NF}$.

Example $4 \mathrm{We}$ want to check whether the knowledge bases $\mathcal{R}_{1}=\{(d f \mid e),(e \bar{f} \mid e)$, $(d e \mid e),(\bar{e} f \vee \bar{e} \bar{f} \mid \mathrm{T}),(\bar{e} \mid \mathrm{T})\}$ over $\Sigma_{1}=\{d, e, f\}$ and $\mathcal{R}_{2}=\{(b a \mid a),(b c \mid a)$, $(a \bar{c} \mid a),(\bar{a} \mid \top)\}$ over $\Sigma_{2}=\{a, b, c\}$ are equivalent under signature renaming and normalization.

Defining $d \lessdot e \lessdot f$ on $\Sigma_{1}$ and $a \lessdot b \lessdot c$ on $\Sigma_{2}$, the results of applying $\Theta^{\rho}$ to both $\mathcal{R}_{1}$ and $\mathcal{R}_{2}$ yields $\Theta^{\rho}\left(\mathcal{R}_{1}\right)=\{(e f \mid d),(d \bar{f} \mid d),(e d \mid d),(\bar{d} f \vee \bar{d} \bar{f} \mid \top),(\bar{d} \mid \top)\}$ and $\Theta^{\rho}\left(\mathcal{R}_{2}\right)=$ $\{(b a \mid a),(b c \mid a),(a \bar{c} \mid a),(\bar{a} \mid \top)\}$. Now we apply $t=\{d \mapsto a, e \mapsto b, f \mapsto c\}$ to $\mathcal{R}_{1}$ and get $t\left(\Theta^{\rho}\left(\mathcal{R}_{1}\right)\right)=\{(b c \mid a),(a \bar{c} \mid a),(b a \mid a),(\bar{a} c \vee \bar{a} \bar{c} \mid \top),(\bar{a} \mid \top)\}$ which is equal under normalization to $\Theta^{\rho}\left(\mathcal{R}_{2}\right)$. Therefore, $\mathcal{R}_{1}$ and $\mathcal{R}_{2}$ are equivalent under signature renaming and normalization.

The second approach renames both knowledge bases to the same signature and then transforms them into $\rho \mathrm{NF}$.

Proposition 12 Let $\Sigma_{1}, \Sigma_{2}$ be two signatures and $(\Sigma, \lessdot)$ be an ordered signature all with the same size. Let $\rho_{1}: \Sigma_{1} \mapsto \Sigma$ and $\rho_{2}: \Sigma_{2} \mapsto \Sigma$ be arbitrary renamings. Let $\mathcal{R}_{1}$ be a knowledge base over $\Sigma_{1}$ and $\mathcal{R}_{2}$ be a knowledge base over $\Sigma_{2}$. Then $\mathcal{R}_{1} \simeq_{v} \mathcal{R}_{2}$ iff $\Theta^{\rho}\left(\rho_{1}\left(\mathcal{R}_{1}\right)\right)={ }_{v} \Theta^{\rho}\left(\rho_{2}\left(\mathcal{R}_{2}\right)\right)$.

Proof This is a consequence of Propositions 9 and 10.

The following example illustrates the second approach. 
Example 5 (Example 4 continued) Based on Proposition 12, we want to check whether the knowledge bases $\mathcal{R}_{1}, \mathcal{R}_{2}$ given in Example 4 are equivalent under signature renaming and normalization. First, we transfer both knowledge bases to the ordered signature $(\Sigma, \lessdot)$ with $\Sigma=\left\{a_{1}, a_{2}, a_{3}\right\}$ and $a_{1} \lessdot a_{2} \lessdot a_{3}$ :

$$
\begin{aligned}
\mathcal{R}_{1} \simeq \mathcal{R}_{1}^{\prime}= & \left\{\left(a_{1} a_{3} \mid a_{2}\right),\left(a_{2} \bar{a}_{3} \mid a_{2}\right),\left(a_{1} a_{2} \mid a_{2}\right),\right. \\
& \left.\left(\bar{a}_{2} a_{3} \vee \bar{a}_{2} \bar{a}_{3} \mid \top\right),\left(\bar{a}_{2} \mid \top\right)\right\} \\
\mathcal{R}_{2} \simeq \mathcal{R}_{1}^{\prime}= & \left\{\left(a_{2} a_{1} \mid a_{1}\right),\left(a_{2} a_{3} \mid a_{1}\right),\left(a_{1} \bar{a}_{3} \mid a_{1}\right),\left(\bar{a}_{1} \mid \top\right)\right\}
\end{aligned}
$$

Applying $\Theta^{\rho}$ yields the two knowledge bases

$$
\begin{aligned}
\Theta^{\rho}\left(\mathcal{R}_{1}^{\prime}\right)= & \left\{\left(a_{2} a_{3} \mid a_{1}\right),\left(a_{1} \bar{a}_{3} \mid a_{1}\right),\left(a_{2} a_{1} \mid a_{1}\right),\right. \\
& \left.\left(\bar{a}_{1} a_{3} \vee \bar{a}_{1} \bar{a}_{3} \mid \top\right),\left(\bar{a}_{1} \mid \top\right)\right\} \\
\Theta^{\rho}\left(\mathcal{R}_{2}^{\prime}\right)= & \left\{\left(a_{2} a_{1} \mid a_{1}\right),\left(a_{2} a_{3} \mid a_{1}\right),\left(a_{1} \bar{a}_{3} \mid a_{1}\right),\left(\bar{a}_{1} \mid \top\right)\right\}
\end{aligned}
$$

which are equivalent under normalization, showing that $\mathcal{R}_{1}$ and $\mathcal{R}_{2}$ are equivalent under signature renaming and normalization.

\section{Reduced antecedentwise normal form}

In addition to the semantic equivalence notion $\equiv_{m o d}$, we will now consider syntactic notions of equivalences of knowledge bases and corresponding normal forms. For comparing or generating knowledge bases, it is useful to abstract from merely syntactic variants. In particular, it is desirable to have minimal versions and normal forms of knowledge bases at hand. The idea of elementwise equivalence [14] is that each piece of knowledge in one knowledge base directly corresponds to a piece of knowledge in the other knowledge base.

Definition 8 (equivalence $\left.\equiv_{e e}[14]\right)$ Let $\mathcal{R}, \mathcal{R}^{\prime}$ be knowledge bases.

- $\mathcal{R}$ is an elementwise equivalent sub-knowledge base of $\mathcal{R}^{\prime}$, denoted by $\mathcal{R} \ll_{e e} \mathcal{R}^{\prime}$, if for every conditional $(B \mid A) \in \mathcal{R}$ that is not self-fulfilling there is a conditional $\left(B^{\prime} \mid A^{\prime}\right) \in \mathcal{R}^{\prime}$ such that $(B \mid A) \equiv\left(B^{\prime} \mid A^{\prime}\right)$.

- $\mathcal{R}$ and $\mathcal{R}^{\prime}$ are strictly elementwise equivalent if $\mathcal{R} \ll_{e e} \mathcal{R}^{\prime}$ and $\mathcal{R}^{\prime} \ll_{e e} \mathcal{R}$.

- $\mathcal{R}$ and $\mathcal{R}^{\prime}$ are elementwise equivalent, denoted by $\mathcal{R} \equiv_{e e} \mathcal{R}^{\prime}$, if either both are inconsistent, or both are consistent and strictly elementwise equivalent.

The knowledge bases $\mathcal{R}_{1}=\{(a \mid \top),(b \mid \top),(a b \mid \top)\}$ and $\mathcal{R}_{2}=\{(a \mid \top),(b \mid \top)\}$ are model equivalent, but not elementwise equivalent since for $(a b \mid \top) \in \mathcal{R}_{1}$ there is no corresponding conditional in $\mathcal{R}_{2}$. The idea of antecedentwise equivalence [17] is to extend elementwise equivalence and to take into account the set of conditionals having the same (or propositionally equivalent) antecedent when comparing to knowledge bases.

Definition $9\left(\operatorname{Ant}(\mathcal{R}), \mathcal{R}_{\mid A}\right.$, ANF [17]) Let $\mathcal{R}$ be a knowledge base.

- $\operatorname{Ant}(\mathcal{R})=\{A \mid(B \mid A) \in \mathcal{R}\}$ is the set of antecedents of $\mathcal{R}$.

- For $A \in \operatorname{Ant}(\mathcal{R})$, the set $\mathcal{R}_{\mid A}=\left\{\left(B^{\prime} \mid A^{\prime}\right) \mid\left(B^{\prime} \mid A^{\prime}\right) \in \mathcal{R}\right.$ and $\left.A \equiv A^{\prime}\right\}$ is the set of $A$-conditionals in $\mathcal{R}$. 
- $\mathcal{R}$ is in antecedent normal form (ANF) if either $\mathcal{R}$ is inconsistent and $\mathcal{R}=\diamond$, or $\mathcal{R}$ is consistent, does not contain any self-fulfilling conditional, contains only conditionals of the form $(A B \mid A)$, and $\left|\mathcal{R}_{|A|}\right|=1$ for all $A \in \operatorname{Ant}(\mathcal{R})$.

Conditionals that are equal up to normalization to $(A B \mid A)$ for some formulae $A, B$ with $A \not \equiv A B$ and $A B \not \equiv \perp$ are called normal form conditionals [23].

Proposition $13(N F C(\Sigma)[23])$ For $N F C(\Sigma)=\left\{(B \mid A) \mid A \subseteq \Omega_{\Sigma}, B \varsubsetneqq A, B \neq \emptyset\right\}$, the set of normal form conditionals over a signature $\Sigma$, the following holds:

(nontrivial) $\quad \operatorname{NFC}(\Sigma)$ does not contain any trivial conditional.

(complete) For every non-trivial conditional over $\Sigma$, there is an equivalent conditional in $\operatorname{NFC}(\Sigma)$.

(minimal) All conditionals in $\mathrm{NFC}(\Sigma)$ are pairwise non-equivalent.

Thus, every knowledge base in ANF is a subset of $N F C(\Sigma)$.

Definition 10 ( $\ll_{a e}$, equivalence $\left.\equiv_{a e}[17]\right)$ Let $\mathcal{R}, \mathcal{R}^{\prime}$ be knowledge bases.

- $\mathcal{R}$ is an antecedentwise equivalent sub-knowledge base of $\mathcal{R}^{\prime}$, denoted by $\mathcal{R} \ll_{a e} \mathcal{R}^{\prime}$, if for every $A \in \operatorname{Ant}(\mathcal{R})$ such that $\mathcal{R}_{\mid A}$ is not self-fulfilling there is an $A^{\prime} \in \operatorname{Ant}\left(\mathcal{R}^{\prime}\right)$ with $\mathcal{R}_{\mid A} \equiv_{\text {mod }} \mathcal{R}_{\mid A^{\prime}}^{\prime}$.

- $\quad \mathcal{R}$ and $\mathcal{R}^{\prime}$ are strictly antecedentwise equivalent if $\mathcal{R} \ll_{a e} \mathcal{R}^{\prime}$ and $\mathcal{R}^{\prime} \ll_{a e} \mathcal{R}$.

- $\mathcal{R}$ and $\mathcal{R}^{\prime}$ are antecedentwise equivalent, denoted by $\mathcal{R} \equiv_{a e} \mathcal{R}^{\prime}$, if either both are inconsistent, or both are consistent and strictly antecedentwise equivalent.

Note that any two inconsistent knowledge bases are also antecedentwise equivalent, e.g., $\{(b \mid a),(\bar{b} \mid a)\} \equiv_{a e}\{(b \mid b),(a \bar{a} \mid \mathrm{T})\}$, thus avoiding cumbersome case distinctions when dealing with consistent and inconsistent knowledge bases. In general, we have:

Proposition 14 ( $\left.\equiv_{a e}[17]\right)$ Let $\mathcal{R}, \mathcal{R}^{\prime}$ be consistent knowledge bases.

1. If $\mathcal{R} \ll_{a e} \mathcal{R}^{\prime}$ then $\operatorname{Mod}\left(\mathcal{R}^{\prime}\right) \subseteq \operatorname{Mod}(\mathcal{R})$.

2. If $\mathcal{R} \equiv_{\text {ae }} \mathcal{R}^{\prime}$ then $\mathcal{R} \equiv_{\text {mod }} \mathcal{R}^{\prime}$.

3. If $\mathcal{R} \ll_{e e} \mathcal{R}^{\prime}$ then $\mathcal{R} \ll_{a e} \mathcal{R}^{\prime}$.

4. If $\mathcal{R} \equiv_{e e} \mathcal{R}^{\prime}$ then $\mathcal{R} \equiv_{a e} \mathcal{R}^{\prime}$.

5. None of the implications (1.) - (4.) holds in general in the reverse direction.

Thus, under antecedentwise equivalence more knowledge bases are equivalent than under elementwise equivalence. For instance, for $\mathcal{R}_{5}=\{(b c \mid a),(c d \mid a)\}$ and $\mathcal{R}_{6}=$ $\{(b d \mid a),(b c d \mid a)\}$ we have $\mathcal{R}_{5} \equiv_{a e} \mathcal{R}_{6}$, but $\mathcal{R}_{5} \not_{e e} \mathcal{R}_{6}$.

While $\equiv_{a e}$ is a better approximation of $\equiv_{m o d}$ than $\equiv_{e e}$, the following example demonstrates a characteristic feature of model equivalence that is not covered by antecedentwise equivalence.

Example 6 (三ae) Let $\mathcal{R}_{7}=\{(b c \mid a),(c \mid a b)\}$ and $\mathcal{R}_{8}=\{(b c \mid a),(b \mid a c)\}$ be knowledge bases. Then $\mathcal{R}_{7} \neq_{a e} \mathcal{R}_{8}$, but $\mathcal{R}_{7} \equiv_{\text {mod }} \mathcal{R}_{8}$.

Furthermore, also for $\mathcal{R}_{7}^{\prime}=\{(a b c \mid a),(a b c \mid a b)\}$ and $\mathcal{R}_{8}^{\prime}=\{(a b c \mid a),(a b c \mid a c)\}$ which are both in ANF, we have $\mathcal{R}_{7}^{\prime} \equiv_{\text {ae }} \mathcal{R}_{8}^{\prime}$, but $\mathcal{R}_{7}^{\prime} \equiv_{\text {mod }} \mathcal{R}_{8}^{\prime}$. 
The knowledge bases $\mathcal{R}_{7}$ and $\mathcal{R}_{8}$ from Example 6 both contain a conditional that is entailed from the other conditionals in that knowledge base using the axioms of system $\mathrm{P}$, i.e., $(c \mid a b)$ in $\mathcal{R}_{7}$ and $(b \mid a c)$ in $\mathcal{R}_{8}$, respectively. Therefore, we now introduce a new normal form prohibiting this kind of redundancy.

Definition 11 (reduced form, RANF) Let $\mathcal{R}$ be a knowledge base.

- $\mathcal{R}$ is in reduced form (with respect to system $\mathrm{P}$ ) if there is no conditional $(B \mid A) \in \mathcal{R}$ such that $A \sim \sim_{\mathcal{R} \backslash(B \mid A)} B$.

- $\mathcal{R}$ is in reduced antecedent normal form $(R A N F)$ if $\mathcal{R}$ is in ANF and in reduced form.

Example $7\left(\equiv_{a e}\right)$ None of the four knowledge bases from Example 6 is in RANF, while, e.g., $\mathcal{R}_{9}=\{(a b c \mid a)\}$ is in RANF. Both $\mathcal{R}_{10}=\{(a c \mid a),(b c \mid b),(a c \vee b c \mid a \vee b)\}$ and $\mathcal{R}_{11}=\{(a c \mid a),(b c \mid b)\}$ are in ANF, but only the obviously simpler knowledge base $\mathcal{R}_{11}$ is in RANF.

Thus, a knowledge base $\mathcal{R}$ in RANF is a most succinct way of expressing the knowledge represented by $\mathcal{R}$ in the sense that there is no proper subset $\mathcal{R}^{\prime}$ of $\mathcal{R}$ licensing the same set of system $\mathrm{P}$ inferences as $\mathcal{R}$. Furthermore, for every consistent knowledge base $\mathcal{R}$, there is a knowledge base $\mathcal{R}^{\prime}$ in RANF that is model equivalent, and hence also system $\mathrm{P}$ inference equivalent to $\mathcal{R}$. This property is shown constructively in the next section

\section{Transforming knowledge bases into ANF and reduced ANF}

In this section, we show how, for any given knowledge base, an equivalent representation in ANF and in RANF can be found. To do so, we present two transformation systems $\Theta^{a}$ and $\Theta^{r a}$ that transform a knowledge base into ANF or RANF, respectively.

The transformation systems utilize a function $\Pi$ with $\Pi(\mathcal{R})=\diamond$ iff $\mathcal{R}$ is inconsistent; $\Pi$ can easily be implemented by the tolerance test for conditional knowledge bases [9]. Using $\Pi$ and the propositional normalization function $v$, the transformation system $\Theta^{a}$ given in Fig. 2 contains four transformation rules:

$(S F) \quad$ removes a self-fulling conditional $(B \mid A)$ with $A \not \equiv \perp$.

$$
\begin{array}{lll}
(S F) \text { self-fulfilling: } & \frac{\mathcal{R} \cup\{(B \mid A)\}}{\mathcal{R}} & A \models B, A \neq \perp \\
(A E) \text { antecedence: } & \frac{\mathcal{R} \cup\left\{(B \mid A),\left(B^{\prime} \mid A^{\prime}\right)\right\}}{\mathcal{R} \cup\left\{\left(B B^{\prime} \mid A\right)\right\}} & A \equiv A^{\prime} \\
(N O) \text { normalization: }: & \frac{\mathcal{R} \cup\{(B \mid A)\}}{\mathcal{R} \cup\{(\nu(A B) \mid \nu(A))\}} & A \neq \nu(A) \text { or } B \neq \nu(A B) \\
(I C) \text { inconsistency: } & \frac{\mathcal{R}}{\diamond} & \mathcal{R} \neq \diamond, \Pi(\mathcal{R})=\diamond
\end{array}
$$

Fig. 2 Transformation rules $\Theta^{a}$ and their applicability conditions for the transformation of knowledge bases into ANF [17]; $\Pi$ is a consistency test, e.g. the tolerance criterion [9], and $v$ a normalization function for propositional formulas 
(AE) merges two conditionals $(B \mid A)$ and $\left(B^{\prime} \mid A^{\prime}\right)$ with propositionally equivalent antecedents to a conditional having this antecedent and the conjunction of the consequents.

(NO) transforms a conditional $(B \mid A)$ by sharpening its consequent to the conjunction with its antecedent and propositionally normalizes the antecedent and the resulting consequent.

(IC) transforms an inconsistent knowledge base into $\diamond$.

By applying the rules in $\Theta^{a}$ exhaustively, every knowledge base can be converted into an antecedentwise equivalent knowledge base in ANF. The transformation system is confluent, i.e., the result is independent of the order in which the rules are applied. For every knowledge base $\mathcal{R}, \Theta^{a}(\mathcal{R})$ is the unique antecedentwise equivalent representation of $\mathcal{R}$ in ANF [23, Proposition 2].

To transform knowledge bases into RANF, we extend $\Theta^{a}$ by another rule:

(RE) removes a conditional $(B \mid A)$ with $A \not \equiv \perp$ from $\mathcal{R}$ if $A$ p-entails $B$ in the context of $\mathcal{R} \backslash\{(B \mid A)\}$.

The complete transformation system $\Theta^{r a}$ is shown in Fig. 3. Its usage is demonstrated in the following example.

Example $8\left(\mathcal{R}_{\text {book }}\right)$ Let $\Sigma_{\text {book }}=\{b, p, t\}$ be a propositional signature where $b$ indicates whether something is a book, $p$ indicates whether something is made out of paper, and $t$ indicates whether something contains text.

The knowledge base $\mathcal{R}_{\text {book }}$ contains five conditionals:

$$
\begin{aligned}
& q_{1}:(p \mid p b) \\
& q_{2}:(p \mid b) \\
& q_{3}:(t \mid b) \\
& q_{4}:(t \mid b p) \\
& q_{5}:(\bar{t} \mid b \bar{p})
\end{aligned}
$$

"Books printed on paper are usually made out of paper."

"Books are usually made out of paper."

"Books usually contain text."

"Books made of paper usually contain text."

"Books that are not made out of paper usually do not contain text."

Now, we apply $\Theta^{r a}$ to $\mathcal{R}_{\text {book }}$.

$$
\begin{array}{lcl}
(R E) \text { reduction: } & \frac{\mathcal{R} \cup\{(B \mid A)\}}{\mathcal{R}} & \Pi((\mathcal{R} \backslash\{(B \mid A)\}) \cup\{(\bar{B} \mid A)\})=\diamond, A \not \equiv \perp \\
(S F) \text { self-fulfilling: } & \frac{\mathcal{R} \cup\{(B \mid A)\}}{\mathcal{R}} & A \models B, A \not \equiv \perp \\
(A E) \text { antecedence: } & \frac{\mathcal{R} \cup\left\{(B \mid A),\left(B^{\prime} \mid A^{\prime}\right)\right\}}{\mathcal{R} \cup\left\{\left(B B^{\prime} \mid A\right)\right\}} & A \equiv A^{\prime} \\
(N O) \text { normalization }: & \frac{\mathcal{R} \cup\{(B \mid A)\}}{\mathcal{R} \cup\{(\nu(A B) \mid \nu(A))\}} & A \neq \nu(A) \text { or } B \neq \nu(A B) \\
(I C) \text { inconsistency: } & \frac{\mathcal{R}}{\diamond} & \mathcal{R} \neq \diamond, \Pi(\mathcal{R})=\diamond
\end{array}
$$

Fig. 3 Transformation rules $\Theta^{r a}$ and their applicability conditions for the transformation of knowledge bases into RANF; $\Pi$ is a consistency test, e.g. the tolerance criterion [9], and $v$ a normalization function for propositional formulas 
$(S F)$ The conditional $q_{1}$ is self-fulfilling. Application of $(S F)$ removes $q_{1}$ from the knowledge base.

$(A E)$ The conditionals $q_{2}$ and $q_{3}$ share the same antecedence. The application of $(A E)$ replaces them with $q_{6}:(p t \mid b)$.

(RE) The knowledge base $\left\{q_{6}, q_{5}\right\}$ p-entails $q_{4}$. Therefore, $q_{4}$ is removed by the application of $(R E)$.

(NO) Normalizing the conditionals $q_{5}$ and $q_{6}$ in the knowledge base yields $q_{5}^{\prime}$ : $(v(\bar{t} b \bar{p}) \mid v(b \bar{p}))$ and $q_{6}^{\prime}:(v(p t b) \mid v(b))$.

By applying $\Theta^{r a}$ exhaustively to $\mathcal{R}_{\text {book }}$, we get the knowledge base $\mathcal{R}_{\text {book }}^{\prime}=$ $\{(v(\bar{t} b \bar{p}) \mid v(b \bar{p})),(v(p t b) \mid v(b))\}$ which is in RANF and model equivalent to $\mathcal{R}_{\text {book }}$.

In contrast to the transformation system $\Theta^{a}$, the system $\Theta^{r a}$ is not confluent.

Example 9 (Non-confluence of $\left.\Theta^{r a}\right)$ Let $\mathcal{R}=\{(v(a b) \mid v(a)),(v(a b) \mid v(b)),(v((a \vee$ $c) d) \mid v(a \vee c)),(v((b \vee c) d) \mid v(b \vee c)),(v(a e f) \mid v(a e))\}$. This knowledge base is already in ANF, but it is not reduced.

The third or the forth conditional can be removed with $(R E)$, yielding either

$$
\mathcal{R}^{\prime}=\{(v(a b) \mid v(a)),(v(a b) \mid v(b)),(v((a \vee c) d) \mid v(a \vee c)),(v(a e f) \mid v(a e))\}
$$

or

$$
\mathcal{R}^{\prime \prime}=\{(v(a b) \mid v(a)),(v(a b) \mid v(b)),(v((b \vee c) d) \mid v(b \vee c)),(v(a e f) \mid v(a e))\}
$$

after exhaustive application of $(N O)$. Neither $\mathcal{R}^{\prime}$ nor $\mathcal{R}^{\prime \prime}$ can be reduced further by $\Theta^{r a}$. Both $\mathcal{R}^{\prime}$ and $\mathcal{R}^{\prime \prime}$ are in RANF, and they are two different normal forms of $\mathcal{R}$ with respect to $\Theta^{r a}$.

Because $\Theta^{r a}$ is not confluent, the result of applying the rules $\Theta^{r a}$ to a knowledge base $\mathcal{R}$ is not uniquely determined in general. In the following, $\Theta^{r a}(\mathcal{R})$ will denote the set of all knowledge bases that can be obtained from $\mathcal{R}$ by exhaustively applying the transformation rules of $\Theta^{r a}$ to $\mathcal{R}$ in some arbitrary order.

Proposition 15 (properties of $\Theta^{r a}$ ) Let $\mathcal{R}$ be a knowledge base, and let $\mathcal{R}^{\prime} \in \Theta^{r a}(\mathcal{R})$.
1. (termination)
$\Theta^{r a}$ is terminating.
2. ( $\equiv_{\text {mod }}$ soundness $)$
$\mathcal{R} \equiv{ }_{\text {mod }} \mathcal{R}^{\prime}$.
3. (consistency)
$\mathcal{R}$ is consistent iff $\Theta^{r a}(\mathcal{R}) \neq\{\diamond\}$.
4. $(R A N F)$
$\mathcal{R}^{\prime}$ is in reduced antecedent normal form.

Proof (1.) The transformation rules $(R E),(S F)$ and $(A E)$ each remove one conditional. $(N O)$ can be applied at most once per conditional, and $(I C)$ can be applied at most once in the whole transformation. Therefore $\Theta^{r a}$ is terminating.

(2.) We will check that $\equiv_{\text {mod }}$ is preserved by every rule in $\Theta^{r a}$. $(R E)$ is applied if $(\mathcal{R} \backslash$ $\{(B \mid A)\}) \cup\{(\bar{B} \mid A)\}$ is inconsistent. In this case $A \sim_{\mathcal{R} \backslash\{(B \mid A)\}} B$, i.e. every model of $\mathcal{R} \backslash$ $\{(B \mid A)\}$ accepts $(B \mid A)$. Therefore removing the conditional $(B \mid A)$ from $\mathcal{R}$ does not affect the set of models.

It was shown that $(S F),(A E),(N O)$, and $(I C)$ preserve model equivalence in [17, Proof of Prop. 2, (三 ${ }_{\text {mod }}$ correctness)]. Therefore, $\mathcal{R} \equiv_{\text {mod }} \mathcal{R}^{\prime}$. 
(3.) Let $\mathcal{R}$ be an inconsistent knowledge base. Property (2.) implies that the application of the rules in $\Theta^{r a}$ to $\mathcal{R}$ cannot lead to a consistent knowledge base. Thus every exhaustive application of rules in $\Theta^{r a}$ will apply $(I C)$ at one point. Therefore $\Theta^{r a}(\mathcal{R})=\{\diamond\}$.

(4.) $\mathcal{R}^{\prime}$ is in ANF, otherwise $(S F),(A E),(N O)$, or $(I C)$ could be applied, and $\mathcal{R}^{\prime}$ is in reduced form, otherwise $(R E)$ could be applied.

Hence, a specific consequence of Proposition 15 is that for every consistent knowledge base $\mathcal{R}$ there is a model equivalent knowledge base $\mathcal{R}^{\prime}$ that is in RANF.

\section{Combining normal forms}

In the previous sections, we deliberately developed and investigated the renaming normal form $\rho \mathrm{NF}$ independently of the normal forms ANF, RANF, and other normal forms that have been proposed for conditional knowledge bases. However, the benefits of $\rho \mathrm{NF}$ can be even better exploited in combination with other normal forms.

An important observation is that renaming has no influence on whether a knowledge base is in ANF or in RANF, respectively, or not.

Proposition 16 Let $R, R^{\prime}$ be knowledge bases such that $\mathcal{R} \simeq \mathcal{R}^{\prime}$.

It holds that:

- $\mathcal{R}$ is in ANF iff $\mathcal{R}^{\prime}$ is in ANF.

- $\mathcal{R}$ is in $R A N F$ iff $\mathcal{R}^{\prime}$ is in $R A N F$.

Proof Both ANF and RANF are defined independent of the symbols in the underlying signature. Changing the symbols by renaming does not affect the normal form.

Combining ANF and RANF with $\rho \mathrm{NF}$, and combining their normal form transformation approaches, we get two new normal forms with corresponding normal form transformations.

Definition 12 ( $\rho$ ANF, $\rho$ RANF, $\left.\Theta^{\rho a}, \Theta^{\rho r a}\right)$ Let $\mathcal{R}$ be a knowledge base.

- $\mathcal{R}$ is in renaming antecedent normal form ( $\rho \mathrm{ANF}$ ) if it is in ANF and in $\rho \mathrm{NF}$. With $\Theta^{\rho a}(\mathcal{R})$, we denote $\Theta^{\rho}\left(\Theta^{a}(\mathcal{R})\right)$.

- $\mathcal{R}$ is in renaming reduced antecedent normal form ( $\rho$ RANF) if it is in RANF and in $\rho$ NF. With $\Theta^{\rho r a}(\mathcal{R})$, we denote $\Theta^{\rho}\left(\Theta^{r a}(\mathcal{R})\right)$.

Two knowledge bases $\mathcal{R}_{1}, \mathcal{R}_{2}$ may have different $\rho$ NFs, but the same $\rho$ ANF or $\rho$ RANF. The latter does not guarantee any more that $\mathcal{R}_{1}$ and $\mathcal{R}_{2}$ are renaming equivalent, but it still ensures that they are model equivalent up to renamings.

Proposition $17\left(\rho \mathrm{ANF}, \Theta^{\rho a}\right)$ Let $\mathcal{R}$ be a knowledge base.

1. (completeness) There is $\mathcal{R}^{\prime}$ in $\rho A N F$ with $\mathcal{R} \simeq$ mod $\mathcal{R}^{\prime}$.

2. (uniqueness) $\quad \Theta^{\rho a}(\mathcal{R})$ is uniquely determined. 

3. $(\rho A N F)$
$\Theta^{\rho a}(\mathcal{R})$ is in $\rho A N F$.
4. $\left(\simeq_{\bmod }\right)$
$\mathcal{R} \simeq$ mod $\Theta^{\rho a}(\mathcal{R})$

Proof We will first show (2.) to (4.).

(2.) Both $\Theta^{\rho}$ and $\Theta^{a}$ yield unique results. Hence, $\Theta^{\rho a}=\Theta^{\rho} \circ \Theta^{a}$ yields unique results as well.

(3.) $\Theta^{a}(\mathcal{R})$ is in ANF. $\Theta^{\rho}\left(\Theta^{a}(\mathcal{R})\right)$ is in $\rho$ NF. Because of Proposition $16, \Theta^{\rho a}(\mathcal{R})=$ $\Theta^{\rho}\left(\Theta^{a}(\mathcal{R})\right)$ is in ANF as well.

(4.) $\mathcal{R} \equiv_{\text {mod }} \Theta^{a}(\mathcal{R})$ and $\Theta^{a}(\mathcal{R}) \simeq \Theta^{\rho}\left(\Theta^{a}(\mathcal{R})\right)=\Theta^{\rho a}(\mathcal{R})$. Therefore, $\mathcal{R} \simeq_{\text {mod }}$ $\Theta^{\rho a}(\mathcal{R})$.

(1.) Because of (2.), there is a unique knowledge base $\Theta^{\rho a}(\mathcal{R})$. Because of (3.), it is in $\rho$ ANF. Because of (4.), $\Theta^{\rho a}(\mathcal{R}) \simeq{ }_{\text {mod }} \mathcal{R}$.

For model equivalence up to renaming of symbols, we get a further property of $\Theta^{\rho a}$.

Proposition $18\left(\Theta^{\rho a}\right)$ For $i=1,2$, let $\mathcal{R}_{i}$ be a knowledge base over $\Sigma_{i}$ and $\rho_{i}: \Sigma_{i} \mapsto \Sigma$ a signature renaming.

Then $\Theta^{\rho a}\left(\rho_{1}\left(\mathcal{R}_{1}\right)\right)=\Theta^{\rho a}\left(\rho_{2}\left(\mathcal{R}_{2}\right)\right)$ implies $\mathcal{R}_{1} \simeq_{\text {mod }} \mathcal{R}_{2}$.

Proof We have

$$
\mathcal{R}_{1} \simeq \rho_{1}\left(\mathcal{R}_{1}\right) \simeq_{\text {mod }} \Theta^{\rho a}\left(\rho_{1}\left(\mathcal{R}_{1}\right)\right)=\Theta^{\rho a}\left(\rho_{2}\left(\mathcal{R}_{2}\right)\right) \simeq_{\text {mod }} \rho_{2}\left(\mathcal{R}_{2}\right) \simeq \mathcal{R}_{2}
$$

because of Proposition 17 (4.).

Thus, two knowledge bases over different signatures are model equivalent up to renamings if they have the same $\rho \mathrm{ANF}$ for some (arbitrary) embeddings into some common signature. Note that Proposition 18 also covers the special case where $\Sigma_{1}=\Sigma_{2}=\Sigma$ and where $\rho_{i}$ is the identity.

Example $10\left(\mathcal{R}_{c a r}\right.$ vs. $\left.\mathcal{R}_{b c f}\right)$ Let $\Sigma_{c a r}=\{c, e, f\}$ and $\mathcal{R}_{c a r}$ as in Example 1 and let $\Sigma_{b c f}=$ $\{b, c, f\}$ and $\mathcal{R}_{b c f}$ as in Example 2. When comparing the knowledge bases $\mathcal{R}_{c a r}$ and $\mathcal{R}_{b c f}$, we observe that their $\rho$ NFs are different and that they are not renaming equivalent. For comparing their $\rho$ ANFs, let us first apply $\Theta^{a}$ to both, yielding:

$$
\begin{aligned}
& \Theta^{a}\left(\mathcal{R}_{c a r}\right)=\{(v(c f) \mid v(f)),(v(c e \bar{f}) \mid v(e)),(v(\bar{e}) \mid v(\top))\} \\
& \Theta^{a}\left(\mathcal{R}_{b c f}\right)=\{(v(b \bar{c} f) \mid v(f)),(v(b c) \mid v(b)),(v(\bar{f}) \mid v(\top))\}
\end{aligned}
$$

Using the ordered standard signature $\Sigma=\{a, b, c\}$ with $a \lessdot b \lessdot c$ and applying $\Theta^{\rho}$, we obtain

$$
\rho A N F_{\Sigma}\left(\mathcal{R}_{c a r}\right)=\rho A N F_{\Sigma}\left(\mathcal{R}_{b c f}\right)=\{(v(a b \bar{c}) \mid v(a)),(v(b c) \mid v(b)),(v(\bar{a}) \mid v(\top))\},
$$

implying $\mathcal{R}_{\text {car }} \simeq_{\text {mod }} \mathcal{R}_{b c f}$.

Specifically, for $\rho(c)=b, \rho(e)=f, \rho(f)=c$, we have $\mathcal{R}_{c a r} \equiv_{\text {mod }} \rho\left(\mathcal{R}_{b c f}\right)$. Because of Proposition 1, we thus have

$$
A \sim_{\mathcal{R}_{c a r}} B \quad \text { iff } \quad \rho^{-1}(A) \sim_{\mathcal{R}_{b c f}} \rho^{-1}(B)
$$

and

$$
\rho(C) \sim_{\mathcal{R}_{c a r}} \rho(D) \quad \text { iff } \quad C \sim_{\mathcal{R}_{b c f}} D
$$

for all formulas $A, B$ and $C, D$ over the signatures of $\mathcal{R}_{c a r}$ and $\mathcal{R}_{b c f}$, respectively. 
In analogy to the properties of $\rho \mathrm{ANF}$ and $\Theta^{\rho a}$, we can show similar properties for $\rho$ RANF and $\Theta^{\rho r a}$.

Proposition $19\left(\rho \mathrm{RANF}, \Theta^{\rho r a}\right)$ Let $\mathcal{R}$ be a knowledge base.

1. (completeness) There is a knowledge base $\mathcal{R}^{\prime}$ in $\rho R A N F$ with $\mathcal{R} \simeq$ mod $\mathcal{R}^{\prime}$.

2. $(\rho R A N F) \quad \Theta^{\rho r a}(\mathcal{R})$ is in $\rho R A N F$.

3. $\left(\simeq_{\text {mod }}\right) \quad \mathcal{R} \simeq$ mod $\Theta^{\operatorname{\rho ra}}(\mathcal{R})$.

4. $\left(\Sigma-\simeq_{\text {mod }}\right) \quad$ For knowledge bases $\mathcal{R}_{1}, \mathcal{R}_{2}$ over $\Sigma_{1}$ and $\Sigma_{2}$, respectively, and signature renamings $\rho_{i}: \Sigma_{i} \mapsto \Sigma$ for $i=1,2$, it holds that $\Theta^{\text {pra }}\left(\rho_{1}\left(\mathcal{R}_{1}\right)\right)=$ $\Theta^{\text {pra }}\left(\rho_{2}\left(\mathcal{R}_{2}\right)\right)$ implies $\mathcal{R}_{1} \simeq_{\text {mod }} \mathcal{R}_{2}$.

Proof We first show (2.) and (3.).

(2.) $\Theta^{r a}(\mathcal{R})$ is in RANF. $\Theta^{\rho}\left(\Theta^{r a}(\mathcal{R})\right)$ is in $\rho$ NF. Because of Proposition $16, \Theta^{\rho r a}(\mathcal{R})=$ $\Theta^{\rho}\left(\Theta^{r a}(\mathcal{R})\right)$ is in RANF as well.

(3.) $\mathcal{R} \equiv_{\text {mod }} \Theta^{r a}(\mathcal{R})$ and $\Theta^{r a}(\mathcal{R}) \simeq \Theta^{\rho}\left(\Theta^{r a}(\mathcal{R})\right)=\Theta^{\rho r a}(\mathcal{R})$. Therefore, $\mathcal{R} \simeq{ }_{\text {mod }}$ $\Theta^{\rho r a}(\mathcal{R})$.

(1.) $\Theta^{\rho r a}$ yields at least one result $\mathcal{R}^{\prime}$. Because of (2.), $\mathcal{R}^{\prime}$ is in $\rho$ ANF. Because of (3.), $\mathcal{R}^{\prime} \simeq_{\text {mod }} \mathcal{R}$.

(4.) $\mathcal{R}_{1} \simeq \rho_{1}\left(\mathcal{R}_{1}\right) \simeq_{\text {mod }} \Theta^{\rho r a}\left(\rho_{1}\left(\mathcal{R}_{1}\right)\right)=\Theta^{\rho r a}\left(\rho_{2}\left(\mathcal{R}_{2}\right)\right) \simeq_{\text {mod }} \rho_{2}\left(\mathcal{R}_{2}\right) \simeq \mathcal{R}_{2}$

Having introduced renaming antecedent normal form $\rho$ RANF, for every knowledge base $\mathcal{R}$, we can use $\Theta^{\rho r a}(\mathcal{R})$ to represent the knowledge expressed by $\mathcal{R}$ and its system $\mathrm{P}$ inferences. $\Theta^{\rho r a}(\mathcal{R})$ is both a smallest knowledge base and it also takes renamings into account.

\section{Generating knowledge bases in $\rho$ RANF}

In the previous sections, for different notions of normal forms, our starting point was a knowledge base $\mathcal{R}$, and we developed methods for transforming $\mathcal{R}$ into these normal forms. In this section, we consider the most succinct normal form, $\rho$ RANF, and look at the "other side of the code". Given a signature $\Sigma$, we address the problem of systematically generating knowledge bases over $\Sigma$ that are in $\rho$ RANF. Specifically, we will introduce two algorithms to generate all consistent knowledge bases in $\rho$ RANF over $\Sigma$. The first algorithm, $K B_{\text {basic }}^{\text {pra }}$, clarifies the basic approach used here to generate knowledge bases. The second algorithm, $K B^{\rho r a}$, yields the same result as $K B_{\text {basic }}^{\text {pra }}$ but is computationally more efficient.

\subsection{Basic algorithm $K B_{\text {basic }}^{\rho r a}$}

$K B_{\text {basic }}^{\text {pra }}($ Algorithm 2) is a basic algorithm to generate consistent knowledge bases in RANF, that are not pairwise equivalent under renaming. To do so, it starts with an empty set of conditionals and tries to extend it by one conditional at a time. We already know that knowledge bases in RANF consist only of normal form conditionals, and we will represent each formula $A \in \mathcal{L}$ uniquely by its set $\Omega_{A}$ of satisfying worlds. Therefore, to generate all knowledge bases in $\rho$ RANF over $\Sigma$, we only have to consider the conditionals in $N F C(\Sigma)=\left\{(B \mid A) \mid A \subseteq \Omega_{\Sigma}, B \varsubsetneqq A, B \neq \emptyset\right\}$ (cf. Proposition 13). 
To avoid generating the same knowledge base multiple times, the algorithm $K B_{\text {basic }}^{\rho r a}$ will add conditionals to a knowledge base in the order of $\preccurlyeq$ as induced by the order $\lessdot$ on $\Sigma$ (cf. Definition 6).

Proposition $20(N F C(\Sigma), \lessdot[23])$ For a linear ordering $\lessdot$ on a signature $\Sigma$, the induced ordering $\leqslant$ according to Definition 5 is a linear ordering on $\mathrm{NFC}(\Sigma)$.

Proof For any two conditionals $\left(A_{1} \mid B_{1}\right),\left(A_{2} \mid B_{2}\right) \in(\mathcal{L} \mid \mathcal{L})_{\Sigma}$ we have $\left(A_{1} \mid B_{1}\right) \lessdot\left(A_{2} \mid B_{2}\right)$, $\left(A_{2} \mid B_{2}\right) \lessdot\left(A_{1} \mid B_{1}\right)$, or both. The last case is only possible if $\left(A_{1} \mid B_{1}\right) \equiv_{\bmod }\left(A_{2} \mid B_{2}\right)$. Because of Proposition 13, two different normal form conditionals cannot be model equivalent. Therefore, $\leqslant$ is a linear order on $\operatorname{NFC}(\Sigma)$.

Note that the symbol $\leqslant$ denotes an order on $\operatorname{NFC}(\Sigma)$ in [23] while it denotes an order on all conditionals in this article; both orders coincide on $N F C(\Sigma)$.

Proposition $21(\operatorname{NFC}(\Sigma)$, $)$ Given a signature $\Sigma$ with linear ordering $\lessdot$, let $[N F C(\Sigma)]_{/ \simeq}=\left\{\left[r_{1}\right]_{\simeq}, \ldots,\left[r_{m}\right]_{\simeq}\right\}$ be the equivalence classes of $N F C(\Sigma)$ induced by renamings such that for each $i \in\{1, \ldots, m\}$, the conditional $r_{i}$ is the canonical conditional

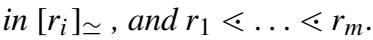

Let $M_{i}=\left[r_{i}\right]_{\simeq} \backslash\left\{r_{i}\right\}$ denote the equivalence class of the canonical normal form conditional $r_{i}$ without $r_{i}$ itself. The canonical ordering $\preccurlyeq$ on $N F C(\Sigma)$ induced by $\lessdot$ follows the schema

$$
r_{1} \prec M_{1} \prec r_{2} \prec M_{2} \prec \ldots \prec r_{m} \prec M_{m} .
$$

and $r \preccurlyeq r^{\prime}$ iff $r \leqslant r^{\prime}$ for all $r, r^{\prime} \in M_{i}$ with $i \in\{1, \ldots, m\}$.

The ordering $\preccurlyeq$ is a linear ordering on $\operatorname{NFC}(\Sigma)$.

Furthermore, its extension to sets of conditionals $\preccurlyeq$ set as given by (3) is a linear ordering on the set of knowledge bases using $\operatorname{NFC}(\Sigma)$.

Proof The structure follows directly from Definition 6 of $\preccurlyeq$. The canonical ordering is a linear ordering on $\operatorname{NFC}(\Sigma)$ as no two normal form conditionals are equivalent (see Proposition 13).

An implication of Proposition 21 is that there is a unique maximum $\max (S)$ in every set $S \subseteq N F C(\Sigma)$ of normal form conditionals with respect to $\preccurlyeq$.

Furthermore, as the conditionals in $\operatorname{NFC}(\Sigma)$ are unique with respect to equivalence under normalization, there is exactly one canonical conditional per equivalence class with respect to equivalence under renaming up to normalization.

Example $11\left(\operatorname{NFC}\left(\Sigma_{a b}\right)\right.$, 飞) While $\operatorname{NFC}\left(\Sigma_{a b}\right)$ with $\Sigma_{a b}=\{a, b\}$ contains 50 conditionals, there are 31 equivalence classes in $\left[\operatorname{NFC}\left(\Sigma_{a b}\right)\right]_{/ \simeq}$; hence $\operatorname{NFC}\left(\Sigma_{a b}\right)$ has 31 canonical normal form conditionals. Note that the ordering defined in Definition 21 differs form the corresponding ordering $\preccurlyeq$ defined in [23] because in [23] all canonical conditionals are $\preccurlyeq$-smaller than all non-canonical conditionals. The complete ordering $\preccurlyeq$ on $N F C\left(\Sigma_{a b}\right)$ is given in Table 1. 
Table 1 The 50 conditionals $r_{01.1} \prec r_{01.2} \prec \ldots \prec r_{30.2} \prec r_{31.1}$ in $N F C\left(\Sigma_{a b}\right)$ for $\Sigma_{a b}=\{a, b\}$, and their 31 equivalence classes [01], .., [31]

\begin{tabular}{|c|c|c|}
\hline Class & First conditional & Second conditional \\
\hline$[01]$ & $r_{01.1}:(\{3\} \mid\{3,2\})$ & $r_{01.2}:(\{3\} \mid\{3,1\})$ \\
\hline$[02]$ & $r_{02.1}:(\{2\} \mid\{3,2\})$ & $r_{02.2}:(\{1\} \mid\{3,1\})$ \\
\hline [03] & $r_{03.1}:(\{3\} \mid\{3,0\})$ & \\
\hline$[04]$ & $r_{04.1}:(\{0\} \mid\{3,0\})$ & \\
\hline$[05]$ & $r_{05.1}:(\{2\} \mid\{2,1\})$ & $r_{05.2}:(\{1\} \mid\{2,1\})$ \\
\hline$[06]$ & $r_{06.1}:(\{2\} \mid\{2,0\})$ & $r_{06.2}:(\{1\} \mid\{1,0\})$ \\
\hline$[07]$ & $r_{07.1}:(\{0\} \mid\{2,0\})$ & $r_{07.2}:(\{0\} \mid\{1,0\})$ \\
\hline$[08]$ & $r_{08.1}:(\{3\} \mid\{3,2,1\})$ & \\
\hline [09] & $r_{09.1}:(\{2\} \mid\{3,2,1\})$ & $r_{09.2}:(\{1\} \mid\{3,2,1\})$ \\
\hline$[10]$ & $r_{10.1}:(\{3,2\} \mid\{3,2,1\})$ & $r_{10.2}:(\{3,1\} \mid\{3,2,1\})$ \\
\hline$[11]$ & $r_{11.1}:(\{2,1\} \mid\{3,2,1\})$ & \\
\hline$[12]$ & $r_{12.1}:(\{3\} \mid\{3,2,0\})$ & $r_{12.2}:(\{3\} \mid\{3,1,0\})$ \\
\hline [13] & $r_{13.1}:(\{2\} \mid\{3,2,0\})$ & $r_{13.1}:(\{1\} \mid\{3,1,0\})$ \\
\hline$[14]$ & $r_{14.1}:(\{0\} \mid\{3,2,0\})$ & $r_{14.1}:(\{0\} \mid\{3,1,0\})$ \\
\hline$[15]$ & $r_{15.1}:(\{3,2\} \mid\{3,2,0\})$ & $r_{15.2}:(\{3,1\} \mid\{3,1,0\})$ \\
\hline$[16]$ & $r_{16.1}:(\{3,0\} \mid\{3,2,0\})$ & $r_{16.2}:(\{3,0\} \mid\{3,1,0\})$ \\
\hline$[17]$ & $r_{17.1}:(\{2,0\} \mid\{3,2,0\})$ & $r_{17.2}:(\{1,0\} \mid\{3,1,0\})$ \\
\hline$[18]$ & $r_{18.1}:(\{2\} \mid\{2,1,0\})$ & $r_{18.2}:(\{1\} \mid\{2,1,0\})$ \\
\hline [19] & $r_{19.1}:(\{0\} \mid\{2,1,0\})$ & \\
\hline [20] & $r_{20.1}:(\{2,1\} \mid\{2,1,0\})$ & \\
\hline [21] & $r_{21.1}:(\{2,0\} \mid\{2,1,0\})$ & $r_{21.2}:(\{1,0\} \mid\{2,1,0\})$ \\
\hline [22] & $r_{22.1}:(\{3\} \mid\{3,2,1,0\})$ & \\
\hline$[23]$ & $r_{23.1}:(\{2\} \mid\{3,2,1,0\})$ & $r_{23.2}:(\{1\} \mid\{3,2,1,0\})$ \\
\hline$[24]$ & $r_{24.1}:(\{0\} \mid\{3,2,1,0\})$ & \\
\hline$[25]$ & $r_{25.1}:(\{3,2\} \mid\{3,2,1,0\})$ & $r_{25.2}:(\{3,1\} \mid\{3,2,1,0\})$ \\
\hline [26] & $r_{26.1}:(\{3,0\} \mid\{3,2,1,0\})$ & \\
\hline [27] & $r_{27.1}:(\{2,1\} \mid\{3,2,1,0\})$ & \\
\hline$[28]$ & $r_{28.1}:(\{2,0\} \mid\{3,2,1,0\})$ & $r_{28.2}:(\{1,0\} \mid\{3,2,1,0\})$ \\
\hline [29] & $r_{29.1}:(\{3,2,1\} \mid\{3,2,1,0\})$ & \\
\hline$[30]$ & $r_{30.1}:(\{3,2,0\} \mid\{3,2,1,0\})$ & $r_{30.2}:(\{3,1,0\} \mid\{3,2,1,0\})$ \\
\hline [31] & $r_{31.1}:(\{2,1,0\} \mid\{3,2,1,0\})$ & \\
\hline
\end{tabular}

Formulas in conditionals are given by sets of worlds, and worlds are represented by their binary number interpretation; e.g., $r_{03.1}:(\{3\} \mid\{3,0\})$ stands for $(\{a b\} \mid\{a b, \bar{a} \bar{b}\})$

Now, we can show the following properties of $K B_{\text {basic }}^{\text {pra }}$.

Proposition $22\left(K B_{\text {basic }}^{\text {pra }}\right)$ Let $\Sigma$ be a signature with a linear ordering $\lessdot$. Then applying $K B_{\text {basic }}^{\text {pra }}$ terminates and returns a set $\mathcal{K B}$ for which the following holds:

1. (correctness)

2. (consistency)
If $\mathcal{R} \in \mathcal{K B}$ then $\mathcal{R}$ is a knowledge base over $\Sigma$. If $\mathcal{R} \in \mathcal{K} \mathcal{B}$ then $\mathcal{R}$ is consistent. 
Algorithm $2 K B_{\text {basic }}^{\text {pra }}-$ Basic algorithm to generate knowledge bases over $\Sigma$ with order $\lessdot$ that are in RANFand $\rho$ NF.

Input: signature $\Sigma$ with order $\lessdot$

Output: set $\mathcal{K B}$ of knowledge bases over $\Sigma$ in reduced antecedentwise normal form (RANF) and in renaming normal form $(\rho \mathrm{NF})$ that are consistent, pairwise not antecedentwise equivalent and pairwise not equivalent under signature renaming

1: $k \leftarrow 0$

2: $L_{0} \leftarrow\{\varnothing\}$

3: while $L_{k} \neq \emptyset$ do

4: $\quad L_{k+1} \leftarrow \emptyset$

5: $\quad$ for $\mathcal{R} \in L_{k}$ do

6: $\quad$ for $r \in N F C(\Sigma)$ do

7: $\quad$ if $\max (\mathcal{R}) \prec r$

8: $\quad$ and $\mathcal{R} \cup\{r\}$ is consistent

9: $\quad$ and $\mathcal{R} \cup\{r\}$ is in RANF

10: $\quad$ and $\mathcal{R} \cup\{r\}$ is in $\rho \mathrm{NF}$

$\triangleright L_{k}$ contains all generated KBs with $k$ conditionals

then

$\triangleright$ For every possible extension...

11:

$L_{k+1} \leftarrow L_{k+1} \cup\{\mathcal{R} \cup\{r\}\}$

$\triangleright$...that is consistent...

$\triangleright$... in RANF...

$\triangleright \ldots$ and in $\rho$ NF...

12: $\quad k \leftarrow k+1$

13:

14: $\operatorname{return} \mathcal{K B} \leftarrow L_{1} \cup \cdots \cup L_{k}$

3. $(R A N F) \quad$ If $\mathcal{R} \in \mathcal{K B}$ then $\mathcal{R}$ is in $R A N F$.

4. $(\rho N F) \quad$ If $\mathcal{R} \in \mathcal{K} \mathcal{B}$ then $\mathcal{R}$ is in $\rho N F$.

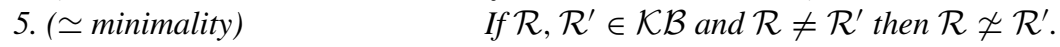

6. (三 ae minimality) $\quad$ If $\mathcal{R}, \mathcal{R}^{\prime} \in \mathcal{K} \mathcal{B}$ and $\mathcal{R} \neq \mathcal{R}^{\prime}$ then $\mathcal{R} \neq_{\text {ae }} \mathcal{R}^{\prime}$.

7. ( $\simeq$ completeness) If $\mathcal{R}$ is a consistent knowledge base in $R A N F$ over $\Sigma$ then there is $\mathcal{R}^{\prime} \in \mathcal{K B}$ and a signature renaming $\varrho$ such that $\mathcal{R}=\varrho\left(\mathcal{R}^{\prime}\right)$.

8. (三 ${ }_{\text {mod }}$ completeness) If $\mathcal{R}$ is a consistent knowledge base over $\Sigma$ then there is $\mathcal{R}^{\prime} \in \mathcal{K} \mathcal{B}$ and a signature renaming $\varrho$ such that $\mathcal{R} \equiv_{\text {mod }} \varrho\left(\mathcal{R}^{\prime}\right)$.

9. ( $\rho R A N F$ completeness) If $\mathcal{R}$ is a consistent knowledge base in $\rho R A N F$ over $\Sigma$ then there is $\mathcal{R}^{\prime} \in \mathcal{K} \mathcal{B}$ such that $\mathcal{R}={ }_{v} \mathcal{R}^{\prime}$.

Proof We prove the stated properties one by one.

(1.) is clear, as the algorithm returns a set of sets of conditionals by design.

(2.) is ensured by the condition in Line 8 .

(3.) is ensured by the condition in Line 9 .

(4.) is ensured by the condition in Line 10 .

(5.) Assume that there are two knowledge bases $\mathcal{R}, \mathcal{R}^{\prime} \in \mathcal{K} \mathcal{B}$ such that $\mathcal{R} \neq \mathcal{R}^{\prime}$ but $\mathcal{R} \simeq \mathcal{R}^{\prime}$. Then (4.) implies that both knowledge bases are in $\rho$ NF. With Proposition 5 it follows that $\mathcal{R}=\mathcal{R}^{\prime}$ because $\mathcal{R} \simeq \mathcal{R}^{\prime}$ and both are in $\rho$ NF. This is a contradiction to the assumption.

(6.) Assume that there are two knowledge bases $\mathcal{R}, \mathcal{R}^{\prime} \in \mathcal{K} \mathcal{B}$ such that $\mathcal{R} \neq \mathcal{R}^{\prime}$ but $\mathcal{R} \equiv \equiv_{\text {ae }} \mathcal{R}^{\prime}$. Because $\mathcal{R}$ and $\mathcal{R}^{\prime}$ are in ANF, the sets $\mathcal{R}_{\mid A}$ and $\mathcal{R}_{\mid A}^{\prime}$ contain at most one element for every antecedent $A$. Therefore, $\mathcal{R} \equiv_{a e} \mathcal{R}^{\prime}$ implies that every conditional in $\mathcal{R}$ 
is equivalent to a conditional in $\mathcal{R}^{\prime}$ and vice versa, i.e. $\mathcal{R} \equiv_{e e} \mathcal{R}^{\prime}$. Because all conditionals in $\mathcal{R}$ and $\mathcal{R}^{\prime}$ are in normal form, this implies $\mathcal{R}=\mathcal{R}^{\prime}$, a contradiction to the assumption.

(7.) For proving (7.), we first prove the following by induction over $k$ :

For $k \geqslant 0$ it holds that every consistent knowledge base $\mathcal{R}$ in RANF and in $\rho \mathrm{NF}$ with $|\mathcal{R}|=k$ is contained in $L_{k}$.

Base case: The empty set is the only knowledge base with 0 conditionals.

Therefore the statement holds for $k=0$, as $L_{0}=\{\emptyset\}$.

Induction step: Let $\mathcal{R}$ be a consistent knowledge base in RANF and $\rho$ NFwith $|\mathcal{R}|=k$. Let $q:=\max (\mathcal{R})$. Then $\mathcal{Q}:=\mathcal{R} \backslash\{q\}$ is in $\rho \mathrm{NF}$ as well (cf. Proposition 7). Moreover, $\mathcal{Q}$ is consistent and in RANF. Hence, the induction hypothesis implies $\mathcal{Q} \in L_{k-1}$. Therefore, $\mathcal{R}=\mathcal{Q} \cup\{q\}$ is added to $L_{k}$ by $K B_{\text {basic }}^{\rho r a}$ because $\max (\mathcal{Q}) \prec q$ and $\mathcal{R}$ is consistent, in RANF, and in $\rho$ NF.

Now let $\mathcal{R}$ be a consistent knowledge base in RANF. With Proposition 5, it follows that there is a renaming $\rho$ and a knowledge base $\mathcal{R}^{\prime}$ in $\rho$ NF with $\mathcal{R}^{\prime}=\rho(\mathcal{R})$. Because $\mathcal{R}$ is consistent and in RANF, $\mathcal{R}^{\prime}$ is consistent and in RANF. With (6) it follows that $\mathcal{R}^{\prime} \in$ $L_{\left|\mathcal{R}^{\prime}\right|} \subseteq \mathcal{K} \mathcal{B}$.

(8.) Let $\mathcal{R}$ be a consistent knowledge base over $\Sigma$. Then there is a knowledge base $\mathcal{R}^{\prime \prime}$ in RANF with $\mathcal{R}^{\prime \prime} \equiv_{\text {mod }} \mathcal{R}$ (cf. Proposition 15). From (7.) it follows that there is a renaming $\rho$ and a knowledge base $\mathcal{R}^{\prime} \in \mathcal{K} \mathcal{B}$ such that $\mathcal{R}^{\prime \prime}=\rho\left(\mathcal{R}^{\prime}\right)$. Therefore, $\mathcal{R} \equiv_{\text {mod }} \rho\left(\mathcal{R}^{\prime}\right)$.

(9.) Let $\mathcal{R}$ be a consistent knowledge base in $\rho$ RANFover $\Sigma$. Property (7.) implies that there is a knowledge base $\mathcal{R}^{\prime} \in \mathcal{K} \mathcal{B}$, such that $\mathcal{R} \simeq \mathcal{R}^{\prime}$, i.e., $\rho N F(\mathcal{R})={ }_{\nu} \rho N F\left(\mathcal{R}^{\prime}\right.$ ) (see Proposition 6). Because both $\mathcal{R}$ and $\mathcal{R}^{\prime}$ are already in $\rho \mathrm{NF}$, it holds that $\mathcal{R}={ }_{v} \mathcal{R}^{\prime}$.

Example 12 Let $\Sigma=\{a, b\}$ and, using the notation as in Table 1,

$$
\begin{aligned}
& \mathcal{R}_{1}=\left\{r_{01.1}:(\{3\} \mid\{3,2\}), r_{01.2}:(\{3\} \mid\{3,1\}), r_{06.1}:(\{2\} \mid\{2,0\})\right\}, \\
& \mathcal{R}_{2}=\left\{r_{01.1}:(\{3\} \mid\{3,2\}), r_{05.2}:(\{1\} \mid\{2,1\})\right\}, \\
& \mathcal{R}_{3}=\left\{r_{01.1}:(\{3\} \mid\{3,2\}), r_{01.2}:(\{3\} \mid\{3,1\}), r_{06.2}:(\{1\} \mid\{1,0\})\right\} .
\end{aligned}
$$

Then $\mathcal{R}_{1}, \mathcal{R}_{2} \in K B_{\text {basic }}^{\text {pra }}(\Sigma)$, but $\mathcal{R}_{3} \notin K B_{\text {basic }}^{\text {pra }}(\Sigma)$ because $\mathcal{R}_{3} \simeq \mathcal{R}_{1}$ and $\mathcal{R}_{1} \prec \mathcal{R}_{3}$.

\subsection{Improved algorithm KB ${ }^{\text {pra }}$}

In order to improve Algorithm $K B_{\text {basic }}^{\rho r a}$ by decreasing its search space, we take a closer look on how to evaluate the conditions in lines 7 to 10 in $K B_{b a s i c}^{\rho r a}$. Similar to the wellknown apriori algorithm determining candidates for extending frequent item sets [24] and also similar to the algorithms introduced in [17, 23] that systematically generate knowledge bases, but in less elaborated normal forms, we can keep track of a set $C$ of conditionals that might be extensions of $\mathcal{R}$.

In this section, we will thus refine $K B_{\text {basic }}^{\text {pra }}$, thereby refining and largely extending the concept of candidates and candidate elimination for knowedge base generation. Furthermore, we employ significant search space improvements for ensuring that all generated knowledge bases are in $\rho \mathrm{NF}$.

Definition 13 (excluded conditionals, $\operatorname{EXC}(r)$ ) Given a signature $\Sigma$, let $r \in N F C(\Sigma)$. The set $\operatorname{EXC}(r)$, called the set of conditionals excluded by $r$, is:

$$
\operatorname{EXC}(r)=\left\{r^{\prime} \in \operatorname{NFC}(\Sigma) \mid r^{\prime} \preccurlyeq r \text { or } \operatorname{ant}(r)=\operatorname{ant}\left(r^{\prime}\right) \text { or }\left\{r, r^{\prime}\right\} \text { is inconsistent }\right\}
$$


Whenever Algorithm $K B_{\text {basic }}^{\text {pra }}$ adds a conditional $r$ to a knowledge base, all conditionals $r^{\prime}$ in $E X C(r)$ can be excluded from the set of candidates for further extensions. If $r^{\prime} \leqslant r$ (or $\left\{r, r^{\prime}\right\}$ is inconsistent or ant $(r)=\operatorname{ant}\left(r^{\prime}\right)$, respectively) the condition in Line 7 (or in Line 8 or in Line 9, respectively) of $K B_{\text {basic }}^{\text {pra }}$ will prohibit the extension of $\mathcal{R} \cup\{r\}$ by $r^{\prime}$.

Note that $\operatorname{EXC}(r)$ is independent of $\mathcal{R}$ and can be pre-computed before running $K B^{\text {pra }}$. For instance, for $r=r_{04.1}:(\{0\} \mid\{3,0\}) \in N F C\left(\Sigma_{a b}\right)$ (cf. Table 1) we have:

$$
\begin{aligned}
\operatorname{EXC}(r)=\left\{r_{01.1}:(\{3\} \mid\{3,2\}), r_{01.2}:(\{3\} \mid\{3,1\}), \ldots, r_{03.1}:(\{3\} \mid\{3,0\}),\right. \\
\left.r_{12.1}:(\{3\} \mid\{3,2,0\}), r_{12.2}:(\{3\} \mid\{3,1,0\}), r_{22.1}:(\{3\} \mid\{3,2,1,0\})\right\}
\end{aligned}
$$

Another improvement regards the check for $\rho \mathrm{NF}$ in Line 10 of Algorithm $K B_{\text {basic }}^{\text {pra }}$. Checking for $\rho \mathrm{NF}$ in Line 10 naively requires to consider all signature renamings over $\Sigma$. For convenience, we will identify the renamings of $\Sigma$ with the set Perm ${ }_{\Sigma}$ of permutations over $\Sigma$, with id being the identity. Thus, there are $\left|\operatorname{Perm}_{\Sigma}\right|=|\Sigma|$ ! many renamings over $\Sigma$. The following proposition provides a criterion for reducing the number of renamings that have to be taken into account if the conditional to be added to $\mathcal{R}$ is $\preccurlyeq$-larger than the maximal conditional in $\mathcal{R}$.

Proposition 23 Let $\mathcal{R}$ be a knowledge base and $r \in N F C(\Sigma)$ such that $\max (\mathcal{R}) \prec r$ and $\mathcal{R} \backslash[r] \simeq$ is in $\rho N F$. Then $\mathcal{R} \cup\{r\}$ is in $\rho N F$ iff for all renamings $\rho$ it holds that:

$$
\rho\left(\mathcal{R} \backslash[r]_{\simeq}\right)=\mathcal{R} \backslash[r]_{\simeq} \text { implies }(\mathcal{R} \cup\{r\}) \cap[r]_{\simeq} \preccurlyeq \rho\left((\mathcal{R} \cup\{r\}) \cap[r]_{\simeq}\right)
$$

Proof Let $\mathcal{R} \backslash[r] \simeq$ be in $\rho$ NF. Let $\mathcal{S}=\mathcal{R} \cup\{r\}$.

Then we have:

$$
\begin{aligned}
& \mathcal{S} \text { in } \rho \mathrm{NF} \\
& \text { iff for every renaming } \rho \text { it holds that } \mathcal{S} \preccurlyeq \rho(\mathcal{S}) \\
& \text { iff for every renaming } \rho \text { it holds that } \mathcal{S} \backslash[r]_{\simeq} \prec \rho\left(\mathcal{S} \backslash[r]_{\simeq}\right) \\
& \quad \text { or } \mathcal{S} \backslash[r]_{\simeq}=\rho\left(\mathcal{S} \backslash[r]_{\simeq}\right) \text { and } \mathcal{S} \cap[r]_{\simeq} \preccurlyeq \rho\left(\mathcal{S} \cap[r]_{\simeq}\right)
\end{aligned}
$$

iff for every renaming $\rho$ it holds that

$$
\mathcal{S} \backslash[r]_{\simeq}=\rho\left(\mathcal{S} \backslash[r]_{\simeq}\right) \quad \text { implies } \quad \mathcal{S} \cap[r]_{\simeq \preccurlyeq} \rho\left(\mathcal{S} \cap[r]_{\simeq}\right)
$$

The equivalence between (8) and (9) holds because a renaming can only map a conditional to another conditional in the same equivalence class (cf. Proposition 2) and the conditionals in $[r] \simeq$ are ordered after the conditionals in $\mathcal{S} \backslash[r] \simeq$ by $\preccurlyeq$. The equivalence between (9) and (10) holds because $\mathcal{S} \backslash[r]_{\simeq}$ is in $\rho \mathrm{NF}$ and therefore, $\mathcal{S} \backslash[r]_{\simeq} \neq \rho\left(\mathcal{S} \backslash[r]_{\simeq}\right)$ implies $\mathcal{S} \backslash[r]_{\simeq} \prec \rho\left(\mathcal{S} \backslash[r]_{\simeq}\right)$.

Proposition 23 implies that for verifying that $\mathcal{R} \cup\{r\}$ is in $\rho \mathrm{NF}$ if $\max (\mathcal{R}) \prec r$ and $\mathcal{R} \backslash[r]_{\simeq}$ is in $\rho \mathrm{NF}$, it is sufficient to check

$$
(\mathcal{R} \cup\{r\}) \cap[r]_{\simeq \preccurlyeq \rho}\left((\mathcal{R} \cup\{r\}) \cap[r]_{\simeq}\right)
$$

for all $\rho \in P:=\{\rho \mid(\mathcal{R} \cup\{r\}) \backslash[r] \simeq=\rho((\mathcal{R} \cup\{r\}) \backslash[r] \simeq)\} \backslash\{$ id $\}$ instead of checking

$$
\mathcal{R} \cup\{r\} \preccurlyeq \rho^{\prime}(\mathcal{R} \cup\{r\})
$$

for all $\rho^{\prime} \in \operatorname{Perm}_{\Sigma}$. 
As conditionals from the same equivalence class are added directly after each other, it is possible to keep track of the set $P$ during the algorithm.

Algorithm $K B^{\rho r a}$ (Algorithm 3 ) is an refined version of Algorithm $K B_{\text {basic }}^{\text {pra }}$ that makes use of these concepts and observations. The set $L_{k}$ contains states instead of knowledge bases. A state is a triple consisting of a knowledge base $\mathcal{R}$, the set $P$ of all signature renamings $\rho$ such that $\rho(\mathcal{R} \backslash E)=\mathcal{R} \backslash E$ where $E:=[\max (\mathcal{R})]_{\simeq}$, and the set $C$ of conditionals that are candidates for the extension of $\mathcal{R}$.

In the initial state, we have $\mathcal{R}=\emptyset, P=\operatorname{Perm}_{\Sigma} \backslash\{$ id $\}$, and $C=\operatorname{NFC}(\Sigma)$.

If a conditional from a new equivalence class is selected, the set $P$ has to be updated. The set $C$ is updated with every extension.

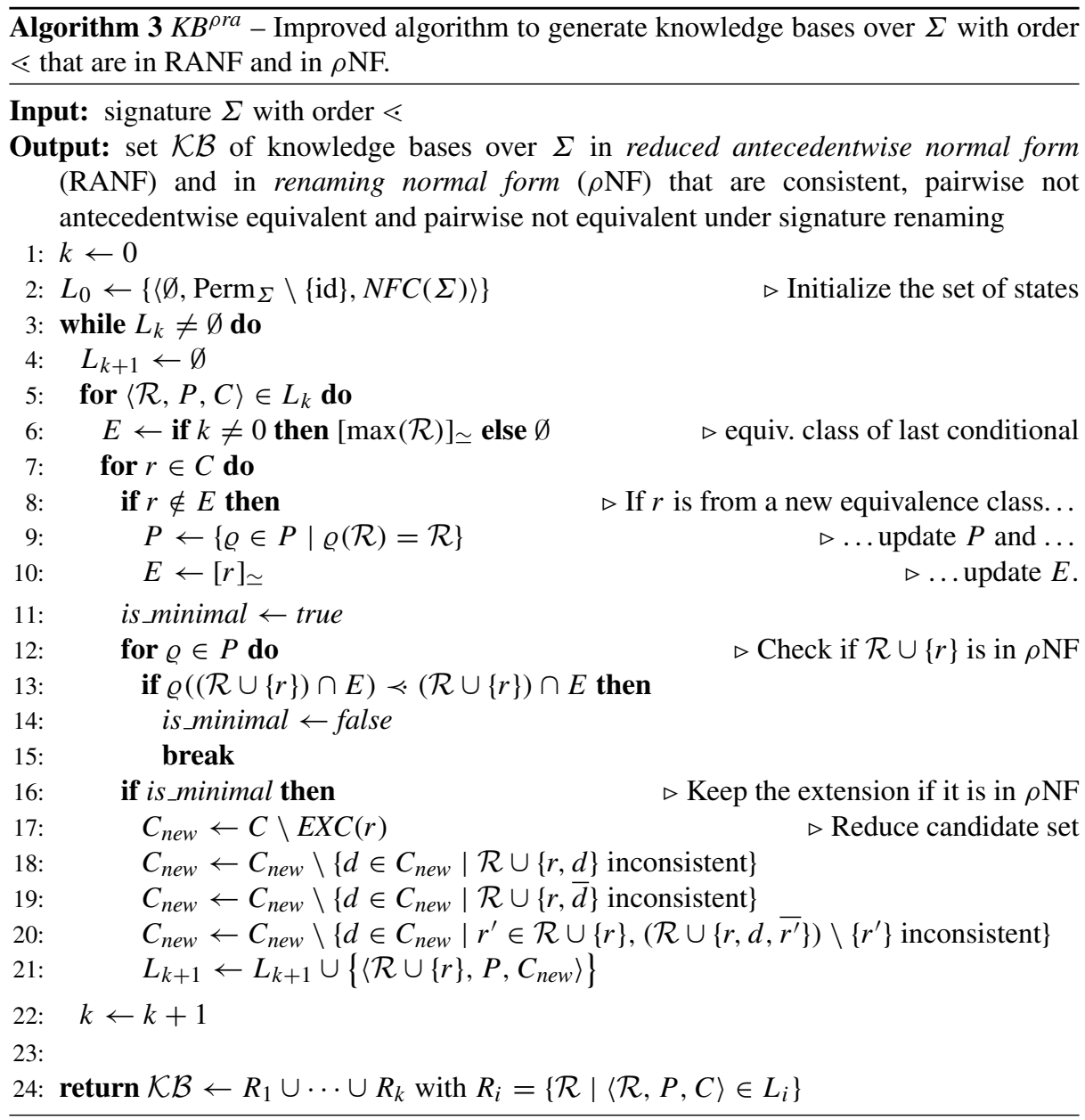

Proposition $24\left(K B^{\rho r a}\right)$ Let $\Sigma$ be a signature with a linear ordering $\lessdot$. Then applying $K B^{\text {pra }}$ terminates and $K B_{\text {basic }}^{\text {pra }}(\Sigma)=K B^{\rho r a}(\Sigma)$. 
Proof There are three differences between the algorithm $K B_{\text {basic }}^{\rho r a}$ and $K B^{\rho r a}$ : The usage of candidate sets, the modified check for $\rho \mathrm{NF}$, and the omission of the checks in Lines 7 to 9 in $K B_{\text {basic }}^{\rho r a}$. In order to prove that $K B^{\rho r a}$ is a correct refinement of $K B_{\text {basic }}^{\rho r a}$, we have to show that

- it is sufficient to consider the conditionals in the candidate set of a knowledge base maintained in $K B^{\rho r a}$ as extensions,

- the check for $\rho \mathrm{NF}$ in $K B^{\rho r a}$ is correct, and

- $\quad$ the checks for $\max (\mathcal{R}) \prec r$, consistency, and RANF done in $K B_{\text {basic }}^{\text {pra }}$ are not necessary due to the filtering of the candidate sets done in $K B^{\rho r a}$.

Let us first consider the candidate sets. Initially, a candidate set contains all normal form conditionals. So far, no possible extension is excluded. Now let us consider the conditionals that are removed from candidate sets in the Lines 17 to 20. Assume the knowledge base $\mathcal{R}$ was just extended by the conditional $r$. The conditionals in $\operatorname{EXC}(r)$ cannot extend $\mathcal{R} \cup\{r\}$ any more by the construction of $\operatorname{EXC}(r)$. If $\mathcal{R} \cup\{r, d\}$ is inconsistent, $d$ cannot extend $\mathcal{R} \cup\{r\}$ or any superset thereof to a consistent knowledge base. Every candidate $d$, such that $\mathcal{R} \cup\{r, \bar{d}\}$ is inconsistent, is an entailment of $\mathcal{R} \cup\{r\}$. Therefore, an extension of $\mathcal{R} \cup\{r\}$ or a any superset thereof by $d$ cannot be in reduced form. Analogously, if $\left(\mathcal{R} \cup\left\{r, d, \overline{r^{\prime}}\right\}\right) \backslash\left\{r^{\prime}\right\}$ is inconsistent for any $r^{\prime} \in \mathcal{R} \cup\{r\}$, then $(\mathcal{R} \cup\{r, d\}) \backslash\left\{r^{\prime}\right\}$ entails $r^{\prime}$. Therefore, $\mathcal{R} \cup\{r, d\}$ or any superset thereof is not in reduced form.

The check for $\rho$ NF in Lines 6 to 15 of $K B^{\rho r a}$ is correct because of Proposition 23.

The checks in Lines 7 to 9 of $K B_{\text {basic }}^{\rho r a}$ were moved to the reduction of the candidate sets. The exclude set $\operatorname{EXC}(r)$ for a conditional $r$ contains all smaller conditionals $r^{\prime} \preccurlyeq r$. All extensions by a single conditional $d$ such that $r$ and $d$ are pairwise inconsistent are already removed by using $E X C(r)$, and all other extensions of $R \cup\{r\}$ that would lead to an inconsistency are removed in Line 18.

The exclude set $E X C(r)$ also contains all conditionals with the same antecedence as $r$, and all conditionals considered here are already normal form conditionals, implying that the extension of a knowledge base with a candidate from the corresponding candidate set is always in ANF. The Lines 19 and 20 ensure that the extension is in reduced form. Therefore the omitted checks from $K B_{\text {basic }}^{\rho r a}$ are not necessary in $K B^{\rho r a}$. In summary, this implies the claim $K B_{\text {basic }}^{\rho r a}(\Sigma)=K B^{\rho r a}(\Sigma)$.

Thus, all properties (1.) - (8.) as stated in Proposition 22 also hold for the set of knowledge base returned by $K B^{\text {pra }}$.

At the same time, $K B^{\rho r a}$ requires less computational effort than $K B_{\text {basic }}^{\rho r a}$, because it uses a significantly reduced search space.

For the check for $\rho \mathrm{NF}$, only the intersection of the equivalence class of the conditional to be added with the knowledge base to be extended has to be checked, and only with respect to the permutations maintained by $K B^{\rho r a}$. For many knowledge bases, this set of permutations still to be taken into account will shrink very fast after a few conditionals have been selected.

Furthermore, the improved algorithm checks unsuccessful extensions less often. If $K B_{\text {basic }}^{\rho r a}$ does not use a conditional in one iteration of the while loop, it will check the same conditional again in the next iteration of the loop. If $K B^{\rho r a}$ does not use a conditional for another reason than the extension not being in $\rho \mathrm{NF}$, it is removed from the candidate set permanently. 


\subsection{Connecting $K B^{\rho r a}$ and $\Theta^{\rho r a}$}

Finally, we can characterize the output of $K B^{\rho r a}$ by utilizing the transformation $\Theta^{\rho r a}$ that transforms knowledge bases to $\rho$ RANF. By applying $\Theta^{\rho r a}$ to every knowledge base over a signature $\Sigma$, we obtain the same set of knowledge bases that is generated by $K B^{\rho r a}$ directly.

Proposition 25 Let $\Sigma$ be a signature with a linear ordering $\lessdot$. Then it holds that

$$
K B^{\rho r a}(\Sigma)=\Theta^{\rho r a}\left(\mathcal{P}\left((\mathcal{L} \mid \mathcal{L})_{\Sigma}\right)\right)
$$

where for a set $S, \mathcal{P}(S)$ denotes the powerset of $S$.

Proof We prove both subset relations of (11).

$\subseteq$ : $\quad$ Let $\mathcal{R} \in K B^{\text {pra }}(\Sigma)$ be a knowledge base. $\mathcal{R}$ is in $\mathcal{P}\left((\mathcal{L} \mid \mathcal{L})_{\Sigma}\right)$. Because $\mathcal{R}$ is in $\rho$ RANF and therefore also a subset of $\operatorname{NFC}(\Sigma)$ (see Proposition 22(3.) and (4.) and Proposition 24), $\Theta^{\rho r a}$ will do nothing but normalize antecedent and consequent. Therefore, $\Theta^{\rho r a}\left(\mathcal{P}\left((\mathcal{L} \mid \mathcal{L})_{\Sigma}\right)\right)$ contains an element that is equal to $\mathcal{R}$ up to normalization.

ㄱ: $\quad$ Let $\mathcal{R} \in \Theta^{\rho r a}\left(\mathcal{P}\left((\mathcal{L} \mid \mathcal{L})_{\Sigma}\right)\right)$ be a knowledge base. Proposition 22(9.) together with Proposition 24 yields that $K B^{\rho r a}(\Sigma)$ contains a knowledge base that is equal to $\mathcal{R}$ up to normalization.

Note the the purpose of Proposition 25 is just to illustrate the interrelationship between normal form transformations on the one hand and systematically generating knowledge bases in normal form on the other hand. Because $\mathcal{L}(\Sigma)$ contains all syntactic variants of each propositional formula over $\Sigma$, there are infinitely many syntactically different knowledge bases in $\mathcal{P}\left((\mathcal{L} \mid \mathcal{L})_{\Sigma}\right)$. Even when restricting the knowledge bases on the right hand side of (11) to normal form conditionals, the number of knowledge bases taken into account in the naive approach of normalizing all corresponding knowledge bases significantly exceeds the number of knowledge bases systematically generated by $K B^{\rho r a}$.

\section{Conclusions and further work}

In this article, we introduced new normal forms for conditional knowledge bases. The semantically motivated reduced antecedent normal form (RANF) takes system Pentailments into account, while the syntax oriented renaming normal form $(\rho \mathrm{NF})$ considers renamings of the underlying signature. The RANF is an improved version of the antecedent normal form (ANF) and further reduces the number of conditionals in a knowledge base.

The transformation system $\Theta^{r a}$ transforms any conditional knowledge base into an equivalent knowledge base in RANF, and the algorithm $\Theta^{\rho}$ transforms a conditional knowledge base into $\rho$ NF. By combining ANF and RANF with $\rho$ NF and combining the corresponding normal form algorithms, we obtained the normal forms $\rho$ ANF and $\rho$ RANF, respectively, and algorithms transforming every knowledge base into any of the newly introduced normal forms.

To systematically generate knowledge bases over a given signature that are in $\rho$ RANF, the most succinct of the normal forms, we developed the algorithm $K B^{\rho r a}$. Furthermore, we proved its key characteristics, i.e., that the generated knowledge bases are consistent, pairwise not antecedentwise equivalent, pairwise not equivalent under signature renaming, and that $K B^{\rho r a}$ is complete because every consistent knowledge base is generated when taking signature renamings and model equivalence into account. 
We are currently working on extending the results of this article in several directions. The ordering $\preccurlyeq$ induced by the underlying ordering $\lessdot$ on the signature is only one of many orderings that could be used in our approach; it could be replaced by another admissible ordering. Furthermore, we will study the normalizing systems $\Theta^{\rho}, \Theta^{r a}, \Theta^{\rho r a}$, and the generating algorithm $K B^{\rho r a}$ with respect to efficiency and complexity. Using implementations of these methods and the InfOCF system [25, 26], we will empirically evaluate properties of given and of systematically generated conditional knowledge bases; some first results of this emperical evaluation are given in [27]. Another open question to be investigated is how the normal forms introduced in this article can be applied or extended to defeasible logics where conditionals occur in the form of defeasible concept subsumptions as in, e.g., [28].

Funding Open Access funding enabled and organized by Projekt DEAL.

Open Access This article is licensed under a Creative Commons Attribution 4.0 International License, which permits use, sharing, adaptation, distribution and reproduction in any medium or format, as long as you give appropriate credit to the original author(s) and the source, provide a link to the Creative Commons licence, and indicate if changes were made. The images or other third party material in this article are included in the article's Creative Commons licence, unless indicated otherwise in a credit line to the material. If material is not included in the article's Creative Commons licence and your intended use is not permitted by statutory regulation or exceeds the permitted use, you will need to obtain permission directly from the copyright holder. To view a copy of this licence, visit http://creativecommons.org/licenses/by/4.0/.

\section{References}

1. Robinson, J.A.: A machine oriented logic based on the resolution principle. J. ACM 12(1), 23-41 (1965)

2. Robinson, J.A., Voronkov, A. (eds.): Handbook of automated reasoning (in 2 volumes). Elsevier and MIT Press (2001)

3. Adams, E.W.: The Logic of Conditionals: An Application of Probability to Deductive Logic. Synthese Library. Springer Science+Business Media, Dordrecht (1975)

4. Lewis, D.: Counterfactuals. Harvard University Press, Cambridge (1973)

5. Kraus, S., Lehmann, D., Magidor, M.: Nonmonotonic reasoning, preferential models and cumulative logics. Artif. Intell. 44, 167-207 (1990)

6. Pearl, J.: System Z: A natural ordering of defaults with tractable applications to nonmonotonic reasoning. In: Parikh, R. (ed.) Proceedings of the 3rd conference on Theoretical aspects of reasoning about knowledge (TARK1990), pp. 121-135. Morgan Kaufmann Publishers Inc., San Francisco (1990)

7. Lehmann, D., Magidor, M.: What does a conditional knowledge base entail? Artif. Intell. 55, 1-60 (1992)

8. Dubois, D., Prade, H.: Conditional objects as nonmonotonic consequence relationships. IEEE Trans. Syst. Man Cybern. Spec. Issue Cond. Event Algebra 24(12), 1724-1740 (1994)

9. Goldszmidt, M., Pearl, J.: Qualitative probabilities for default reasoning, belief revision, and causal modeling. Artif. Intell. 84, 57-112 (1996)

10. Benferhat, S., Dubois, D., Prade, H.: Possibilistic and standard probabilistic semantics of conditional knowledge bases. J. Log. Comput. 9(6), 873-895 (1999)

11. Kern-Isberner, G.: Conditionals in nonmonotonic reasoning and belief revision. LNAI, vol. 2087. Springer, Berlin (2001)

12. Kern-Isberner, G.: A thorough axiomatization of a principle of conditional preservation in belief revision. Ann. Math. Artif. Intell. 40(1-2), 127-164 (2004)

13. Beierle, C., Eichhorn, C., Kern-Isberner, G., Kutsch, S.: Properties and interrelationships of skeptical, weakly skeptical, and credulous inference induced by classes of minimal models. Artif. Intell. 297. https://doi.org/10.1016/j.artint.2021.103489. (in press, online 2 Mar 2021) (2021)

14. Beierle, C., Eichhorn, C., Kern-Isberner, G.: A transformation system for unique minimal normal forms of conditional knowledge bases. In: ECSQARU 2017, LNCS 10369, pp. 236-245. Springer, Berlin (2017)

15. Beierle, C., Eichhorn, C., Kern-Isberner, G.: On transformations and normal forms of conditional knowledge bases. In: Benferhat, S., Tabia, K., Ali, M. (eds.) Advances in Artificial Intelligence: From Theory to Practice, LNCS, vol. 10350, pp. 488-494. Springer (2017) 
16. Beierle, C.: Inferential equivalence, normal forms, and isomorphisms of knowledge bases in institutions of conditional logics. In: Hung, C.-C., Papadopoulos, G.A. (eds.) The 34th ACM/SIGAPP Symposium on Applied Computing (SAC '19), April 8-12, 2019, Limassol, Cyprus, pp. 1131-1138. ACM, New York (2019)

17. Beierle, C., Kutsch, S.: On the antecedent normal form of conditional knowledge bases. In: KernIsberner, G., Ognjanović, Z. (eds.) Symbolic and Quantitative Approaches to Reasoning with Uncertainty - 15th European Conference, ECSQARU 2019, Lecture Notes in Computer Science, vol. 11762, pp. 175-186. Springer (2019)

18. Goguen, J., Burstall, R.: Institutions: Abstract model theory for specification and programming. J. ACM 39(1), 95-146 (1992)

19. Beierle, C., Haldimann, J.: Normal forms of conditional knowledge bases respecting system Pentailments. In: Herzig, A., Kontinen, J. (eds.) FoIKS 2020, LNCS, vol. 12012, pp. 22-41. Springer (2020)

20. Beierle, C., Haldimann, J.: Transforming conditional knowledge bases into renaming normal form. In: Barták, R., Bell, E. (eds.) Proceedings of the 33rd International Florida Artificial Intelligence Research Society Conference, FLAIRS 2020, pp. 563-568. AAAI Press (2020)

21. Spohn, W.: The Laws of Belief: Ranking Theory and Its Philosophical Applications. Oxford U. Press, Oxford (2012)

22. Pearl, J.: Probabilistic reasoning in intelligent systems. Morgan Kaufmann, San Mateo (1988)

23. Beierle, C., Kutsch, S.: Systematic generation of conditional knowledge bases up to renaming and equivalence. In: Calimeri, F., Leone, N., Manna, M. (eds.) Logics in Artificial Intelligence - 16th European Conference, JELIA 2019, Rende, Proceedings, LNAI, vol. 11468, pp. 279-286. Springer (2019)

24. Agrawal, R., Mannila, H., Srikant, R., Toivonen, H., Verkamo, A.I.: Fast discovery of association rules. In: Fayyad, U.M., Piatetsky-Shapiro, G., Smyth, P., Uthurusamy, R. (eds.) Advances in knowledge discovery and data mining, pp. 307-328. MIT Press, Cambridge (1996)

25. Beierle, C., Eichhorn, C., Kutsch, S.: A practical comparison of qualitative inferences with preferred ranking models. KI - Künstliche Intell. 31(1), 41-52 (2017)

26. Kutsch, S.: InfOCF-Lib: A Java library for OCF-based conditional inference. In: Beierle, C., Ragni, M., Stolzenburg, F., Thimm, M. (eds.) Proceedings of the 8th Workshop on Dynamics of Knowledge and Belief (DKB-2019) and the 7th Workshop KI \& Kognition (KIK-2019) co-located with 44nd German Conference on Artificial Intelligence (KI 2019), CEUR Workshop Proceedings, vol. 2445, pp. 47-58. CEUR-WS.org, Kassel (2019)

27. Beierle, C., Haldimann, J., Kutsch, S.: A complete map of conditional knowledge bases in different normal forms and their induced system P inference relations over small signatures. In: Proceedings of the 34th International Florida Artificial Intelligence Research Society Conference, FLAIRS 2021 (2021)

28. Britz, K., Casini, G., Meyer, T., Varzinczak, I.: A KLM perspective on defeasible reasoning for description logics. In: Lutz, C., Sattler, U., Tinelli, C., Turhan, A.-Y., Wolter, F. (eds.) Description Logic, Theory Combination, and All That - Essays Dedicated to Franz Baader on the Occasion of His 60th Birthday, LNCS, vol. 11560, pp. 147-173. Springer (2019)

Publisher's note Springer Nature remains neutral with regard to jurisdictional claims in published maps and institutional affiliations. 